\title{
Robots State Estimation and Observability Analysis Based on Statistical Motion Models
}

\author{
Wei $\mathrm{Xu}^{1}$, Dongjiao $\mathrm{He}^{1}$, Yixi Cai ${ }^{1}$, Fu Zhang ${ }^{1}$
}

\begin{abstract}
This paper presents a generic motion model to capture mobile robots' dynamic behaviors (translation and rotation). The model is based on statistical models driven by white random processes and is formulated into a full state estimation algorithm based on the error-state extended Kalman filtering framework (ESEKF). Major benefits of this method are its versatility, being applicable to different robotic systems without accurately modeling the robots' specific dynamics, and ability to estimate the robot's (angular) acceleration, jerk, or higherorder dynamic states with low delay. Mathematical analysis with numerical simulations are presented to show the properties of the statistical model-based estimation framework and to reveal its connection to existing low-pass filters. Furthermore, a new paradigm is developed for robots observability analysis by developing Lie derivatives and associated partial differentiation directly on manifolds. It is shown that this new paradigm is much simpler and more natural than existing methods based on quaternion parameterizations. It is also scalable to high dimensional systems. A novel thin set concept is introduced to characterize the unobservable subset of the system states, providing the theoretical foundation to observability analysis of robotic systems operating on manifolds and in high dimension. Finally, extensive experiments including full state estimation and extrinsic calibration (both POS-IMU and IMU-IMU) on a quadrotor UAV, a handheld platform and a ground vehicle are conducted. Comparisons with existing methods show that the proposed method can effectively estimate all extrinsic parameters, the robot's translation/angular acceleration and other state variables (e.g., position, velocity, attitude) of high accuracy and low delay.
\end{abstract}

\section{INTRODUCTION}

Online estimation of high-order dynamics states (e.g., translational and angular acceleration) is fundamentally important for many robots and robotic techniques, such as control [1][4], disturbance estimation [5]-[9], model identification [10][12], trajectory generation [13], and extrinsic calibration: in robot's control, it is often necessary to measure or estimate the forces (or accelerations) and torques (or angular accelerations) applied on the robots in order to compute the next control action, estimate the external disturbances, or identify an UAV's aerodynamic parameters; In the calibration of multiple IMUs, angular accelerations are picked up by different accelerometers and need to be accurately estimated for estimating the extrinsic parameters of different IMUs.

IMUs have been widely used in robotics to measure the robots' acceleration and angular velocity [14]. However, raw IMU measurements typically suffer from various imperfections, such as bias and noise, and cannot be fed to a controller directly. In fact, in robotics community, accelerometers are mainly used to provide attitude (and/or position and velocity)

\footnotetext{
${ }^{1}$ All authors are with Department of Mechanical Engineering, University of Hong Kong. \{xuweii, hdj65822, Yixicai, fuzhang\}@hku.hk
}

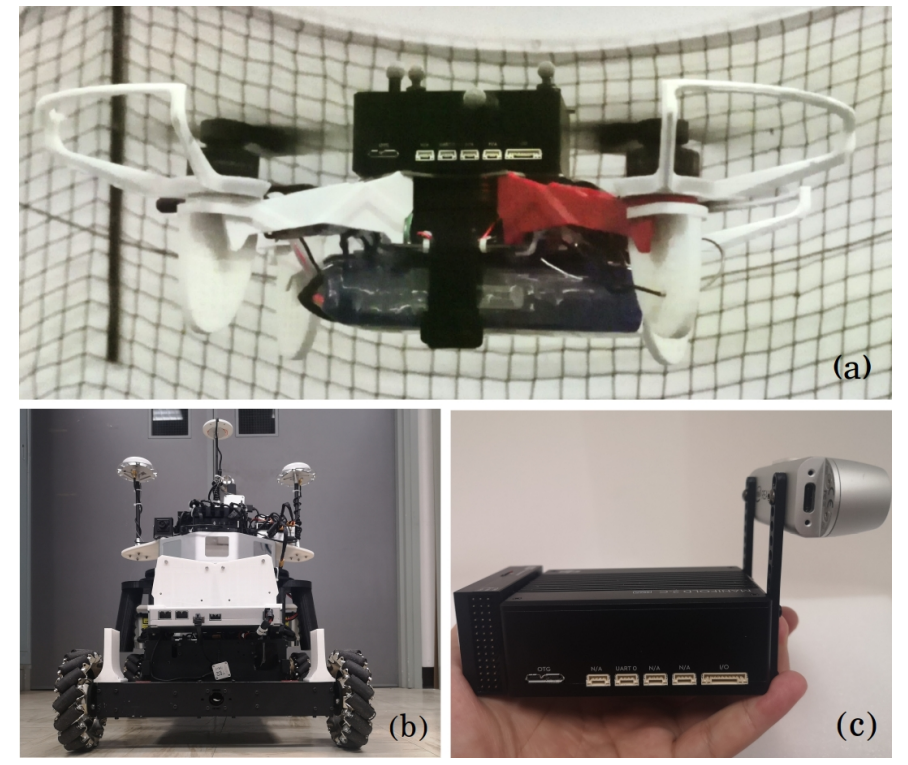

Fig. 1. Our proposed method is able to estimate the kinematics state (i.e., position, velocity, and attitude), dynamics state (e.g., (angular) translation, jerk, etc.), and extrinsic parameters simultaneously and online and is applicable to a variety of mobile platforms, including: (a) Unmanned Aerial Vehicles (UAVs); (b) Unmanned Ground Vehicles (UGVs); (c) Handheld compact IMU-CameraComputer module.

estimation. To name a few, Mahony et al. $[15,16]$ proposed a nonlinear complementary filter operating on the special orthogonal group $S O(3)$ for UAVs' attitude estimation using IMUs. This filter is easy to implement and computationally cheap but is sub-optimal due to the constant feedback gain. Smith and Schmidt [17] firstly used the extended Kalman filter (EKF) for spacecraft attitude and velocity estimation based on IMU measurements. Since the standard EKF works in Euclidean space while a robot's attitude is naturally on $S O(3)$, this discrepancy has motivated the error state EKF (ESEKF) [18]-[20], which workaround the singularity problem caused by minimal parameterization of $S O(3)$ by updating the error attitude [21]. Lu et al. [22] further considered narrow-band noises in IMU-based attitude estimation.

Besides attitude estimation, IMUs are popularly combined with other sensors such as GPS [23], motion capture systems [24], cameras [25]-[27], LiDARs [28, 29], optical flow [30], Pitot tube [31] within an EKF or unscented Kalman filtering (UKF) framework. This closely-coupled estimation method has been proved to be very effective in estimating the robot's kinematic states including position, velocity, attitude, and IMU bias. The multi-sensor fusion approach has also motivated extrinsic calibration: offline methods include maximum likelihood (ML) [32], pose graph optimization [25], EKF 
smoother [12]; online methods typically view the extrinsic parameter as a state and use the same EKF/UKF formulation as in state estimation to additionally estimate the extrinsic parameters [33]-[35].

A major problem of the above works is the absence of highorder dynamics states (e.g., acceleration) estimation. This paper presents a generic model to capture the dynamic behaviors of a variety of mobile robots. The key idea is to model a robot's translational and angular dynamics by a statistical model with tunable covariance. Based on this generic model, we formulate a new error state EKF framework that can additionally estimate the robot high-order dynamics states such as acceleration that are not capable by normal EKF [21]. Mathematical analysis are conducted to show its stability and reduced phase delay when compared to linear filtering techniques. To investigate the observability of the proposed formulation, we explicitly represent the nonlinear observability rank condition on the manifold $S O(3)$, leading to simpler, scalable, and more natural observability analysis than existing quaternion-based analysis. The formulated algorithm is finally verified by both simulations and extensive experiments on real robots. Results show that it is able to estimate the kinematic states (i.e., position, attitude, velocity, IMU biases), dynamics states (e.g., acceleration), and extrinsic parameters (e.g., POS-IMU and IMU-IMU) in real time and for a variety of moving platforms, including UAVs, ground vehicles and handheld devices. Moreover, the proposed method delivers estimation performance comparable to normal EKFs [21] for kinematics states while in the meantime achieves low latency dynamics states estimation comparable to non-causal zero-phase filters [36].

The rest of the paper is organized as follows: Section II summarizes the related works of states estimation, statistical dynamics model and observability analysis in robotics researches; Section III introduces and analyzes the statistical motion model, based on which the error-state EKF is detailed in Section IV; Observability analysis is presented in Section V; and Section VI and VII presents simulation and experiment results, respectively; Finally, Section VIII draws conclusions.

\section{RELATED WORK}

In existing methods for robotic states estimation, such as GPS/IMU navigation [21], visual IMU navigation and online calibration [25]-[27, 34, 35, 37, 38], and LiDAR/IMU navigation [28], the acceleration and angular velocity are solved from the IMU measurements and are then substituted into kinematics model to form a system "driven" by the known IMU measurement. This enables to estimate the kinematic states, such as position, velocity, attitude and IMU bias, but the acceleration and angular velocity themselves are viewed as input to the kinematic model and their values are not filtered. To do so, an ad-hoc linear filter (e.g., Butterworth filter) is usually required to reduce the noises in accelerometer measurements or gyro measurements. This, however, causes considerable output delay due to the Bode's relations [39]. Another possible approach is to carefully model the robot dynamics and invert it to obtain the forces or torques from the robot outputs, such as the disturbance observers used in [5]$[9,40]$. This dynamic model could also be used with an
EKF or UKF to estimate the robot (angular) acceleration such as in [11, 33]. Nevertheless, such a model-based approach needs an accurate model of the robot dynamics which are often subject to various uncertainties, such as motor delay, unmodeled internal dynamics, etc. Instead of using a first principle dynamic model, we propose to use a statistical model to capture the robot dynamics. This statistical model is augmented with the kinematic model where the acceleration appears as a state (instead of input) to the system, enabling itself to be estimated by EKFs. The proposed method leads to lower estimation delay than ad-hod filters and is more generic and robust than model-based methods [11, 33].

Modeling the unknown inputs as a statistical model has been exploited in some prior work and proved effective in practice, such as the Brownian motion for modeling the angular and translational acceleration in both visual-inertial navigation [41] and vision-only navigation [42]. A even simpler constant velocity model driven by a random noise were also used in [43, 44]. Besides the state estimation in robotics, Hoff et al. [12] used a quadratic integration stochastic process to model an aircraft's acceleration which enables to identify its aerodynamics. These works usually considered a specific (and usually low order) statistical model and ignored their effect to the system observability. This paper proposes a generic statistical model characterized by its power spectral density and proves that it does not affect the overall observability. Moreover, a new concept, thin subset, is introduced to characterize the unobservable space of the augmented states.

Observability plays an important role in robotic states estimation [45]. A well-known criterion for nonlinear system observability is the observability rank condition based on Lie derivatives [46]. This criterion has been widely used in robotics to determine the observability of a robotic system, such as range sensor-based 2D localization [47, 48], visualinertial navigation $[49,50]$, online camera-IMU extrinsic calibration [34, 35], and online parameter identification [33, 51], etc. These works usually represent the attitude as a quaternion $\mathbf{q}$ and view it as a flat vector in $\mathbb{R}^{4}$ to calculate the Lie derivatives required in the observability rank condition. To compensate for the over-parameterization when using quaternion, an additional virtual measurement $\mathbf{q}^{T} \mathbf{q}=1$ is added. Although this method correctly determines the system observability, it undermines the structure of the manifold $S O(3)$. Moreover, the observability analysis is considerably complicated due to the over-parameterization. In this paper, we show that this over-parameterization is totally unnecessary and can be completely avoided by developing Lie derivatives directly on $S O(3)$. Besides preserving the manifold structure, the new implementation leads to a much simpler and more scalable observability analysis.

\section{Statistical Motion Model}

\section{A. Motion model}

The dynamics states (e.g., translational acceleration, angular acceleration) of a robotic system usually depends on the control actions and/or other forces and torques from the 
environment. In scenarios such as state estimation of human handheld devices or a UAV intruder, the control actions that drive the system are typically not known to the estimator. Even when such information is available (e.g., estimation of the robot itself states), the dynamic model from the control actions to the state usually suffers from significant uncertainties and/or is affected by environmental disturbances.

We propose to model the dynamics state $\mathbf{u}$ as a colored stochastic process shown below.

$$
\begin{aligned}
\dot{\gamma} & =\mathbf{A}_{\boldsymbol{\gamma}} \boldsymbol{\gamma}+\mathbf{B}_{\boldsymbol{\gamma}} \mathbf{w}_{\boldsymbol{\gamma}} \\
\mathbf{u} & =\mathbf{C}_{\boldsymbol{\gamma}} \boldsymbol{\gamma}
\end{aligned}
$$

where $\left(\mathbf{C}_{\boldsymbol{\gamma}}, \mathbf{A}_{\gamma}\right)$ is observable and $\left(\mathbf{A}_{\gamma}, \mathbf{B}_{\gamma}\right)$ is controllable, $\mathbf{w}_{\boldsymbol{\gamma}} \sim \mathcal{N}\left(0, \mathcal{Q}_{\boldsymbol{\gamma}}\right)$ are zero mean Gaussian white noises. The resultant output $\mathbf{u}$ from (1) has a power spectral density (PSD) function $\mathbf{S}_{\mathbf{u u}}(\omega)=\mathbf{G}_{\boldsymbol{\gamma}}(j \omega) \mathcal{Q}_{\boldsymbol{\gamma}} \mathbf{G}_{\boldsymbol{\gamma}}(-j \omega)^{T}$, where $\mathbf{G}_{\boldsymbol{\gamma}}(j \omega)=$ $\mathbf{C}_{\boldsymbol{\gamma}}\left(j \omega \mathbf{I}-\mathbf{A}_{\boldsymbol{\gamma}}\right)^{-1} \mathbf{B}_{\boldsymbol{\gamma}}$.

We posit that the dynamic behaviors of a system (possibly with internal feedback controllers) can be described, statistically, by a certain random process. While it sounds less intuitive as a system is usually designed to accomplish certain tasks and its behaviors are mostly deterministic instead of random, deterministic behavior can always be viewed as a sample of a collection or ensemble of signals (i.e., random process) [52]. With this hypothesis, the model parameters (i.e., $\mathbf{A}_{\gamma}, \mathbf{B}_{\gamma}, \mathbf{C}_{\gamma}$ and $\mathcal{Q}_{\gamma}$ ) should be chosen such that the resultant PSD $\mathbf{S}_{\mathbf{u u}}(\omega)$ agrees with the actual state PSD. Then, the state distribution can be effectively captured by (1) while their exact value can be inferred from the measured data.

Since the motion of robotic systems usually possesses certain smoothness (e.g., due to actuator delay), quick changes in accelerations are relatively unlikely and a low-pass type $\mathbf{G}_{\boldsymbol{\gamma}}(j \omega)$ usually suffices. More specifically, we propose to use an $n$-th order integrator as shown in (2). A benefit of the integrator is its sparse structure, which considerably saves the computation.

$$
\begin{aligned}
\underbrace{\left[\begin{array}{c}
\dot{\gamma}_{1} \\
\vdots \\
\dot{\gamma}_{N}
\end{array}\right]}_{\dot{\gamma}} & =\underbrace{\left[\begin{array}{cccc}
0 & 1 & & \\
& \ddots & \ddots & \\
& & 0 & 1 \\
& & & 0
\end{array}\right]}_{\mathbf{A}_{\boldsymbol{\gamma}}} \underbrace{\left[\begin{array}{c}
\gamma_{1} \\
\vdots \\
\gamma_{N}
\end{array}\right]}_{\boldsymbol{\gamma}}+\underbrace{\left[\begin{array}{c}
w_{\gamma_{1}} \\
\vdots \\
w_{\gamma_{N}}
\end{array}\right]}_{\mathbf{w}_{\gamma}} \\
u & =\underbrace{\left[\begin{array}{llll}
1 & 0 & \ldots & 0
\end{array}\right]}_{\mathbf{C}_{\boldsymbol{\gamma}}} \gamma
\end{aligned}
$$

where $w_{\gamma_{i}} \sim \mathcal{N}\left(0, q_{\gamma_{i}}\right)$. The resultant PSD is:

$$
S_{u u}(\omega)=\sum_{i=1}^{N} \frac{q_{\gamma_{i}}}{\omega^{2 i}}
$$

where $q_{\gamma_{i}}$ are tunable parameters. Fig. 2 shows the case when $N=4$ and $q_{\gamma_{i}}=1$.

Remark: The integrator model in (2) has been used in some applications, such as the constant velocity model (i.e., $N=1$ ) used in SLAM [43] and the quadratic stochastic process (i.e., $N=4)$ used in the aircraft model identification [53, 54].

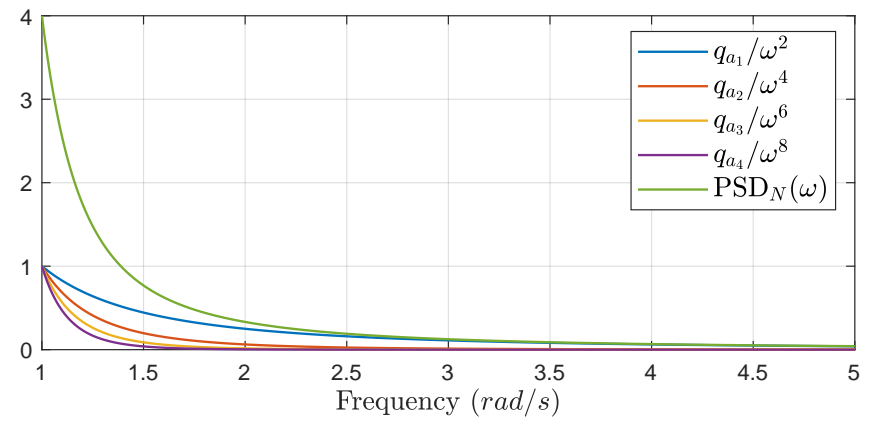

Fig. 2. The output PSD of colored stochastic when $N=4$ and $q_{\gamma_{i}}=1$.

\section{B. System observability}

We analyze the property of the proposed motion model when it is combined with the system kinematic model. To simplify the analysis, we use a linear kinematic model while defer the analysis for real nonlinear robotic systems to Section $\mathrm{V}$. Assume the kinematic model is

$$
\begin{aligned}
\dot{\mathbf{s}} & =\mathbf{A}_{\mathbf{s}} \mathbf{s}+\mathbf{B}_{\mathbf{s}} \mathbf{u} \\
\mathbf{y}_{\mathbf{s}} & =\mathbf{C}_{\mathbf{s}} \mathbf{s}+\mathbf{n}_{\mathbf{s}}
\end{aligned}
$$

where $\mathbf{u}$ (i.e., acceleration) is the dynamics state from the motion model (1). This model is assumed to be a minimal realization and the corresponding transfer function is $\mathbf{G}_{\mathbf{s}}(j \omega)=\mathbf{C}_{\mathbf{s}}\left(j \omega \mathbf{I}-\mathbf{A}_{\mathbf{s}}\right)^{-1} \mathbf{B}_{\mathbf{s}}$.

Concatenating the motion model (1) with (3) in series leads to a system in Fig. 3, where the output is the original system output $\mathbf{y}_{s}$. The output could also contain dynamics state measurements $\mathbf{u}_{m}$. For example, when estimating the state of the robot itself, the acceleration is usually measured by an onboard accelerometer. In this case, $\mathbf{u}_{m}=\mathbf{u}+\mathbf{n}_{u}$ where $\mathbf{n}_{u}$ is the measurement noise.

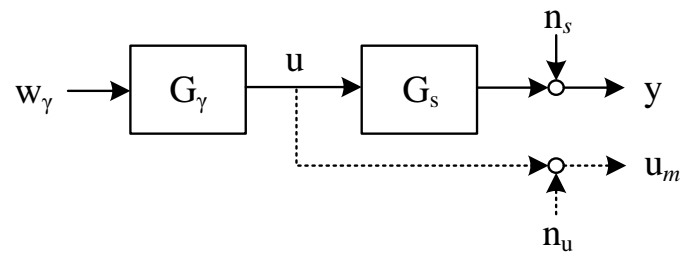

Fig. 3. The concatenated system consists of a colored stochastic process $\mathbf{G}_{\gamma}$ and kinematic model $\mathbf{G}_{\mathbf{s}}$.

On the one hand, When there is no measurement $\mathbf{u}_{m}$, the concatenated system $\mathbf{G}=\mathbf{G}_{\mathbf{s}}(j \omega) \mathbf{G}_{\boldsymbol{\gamma}}(j \omega)$ is observable if there is no pole zero cancellation between $\mathbf{G}_{\mathbf{s}}(j \omega)$ and $\mathbf{G}_{\boldsymbol{\gamma}}(j \omega)$. On the other hand, when $\mathbf{u}_{m}$ is present, the concatenated system is always observable (see Lemma 1 below). In either case, once the system is observable, a Kalman filter could be employed to estimate the dynamics state $\mathbf{u}$ from the system output $\mathbf{y}_{s}$ (and state measurement $\mathbf{u}_{m}$ ).

Lemma 1. For any two linear observable systems 
$\left(\mathbf{C}_{\mathbf{s}}, \mathbf{A}_{\mathbf{s}}, \mathbf{B}_{\mathbf{s}}\right)$ and $\left(\mathbf{C}_{\gamma}, \mathbf{A}_{\gamma}, \mathbf{B}_{\gamma}\right)$, their series concatenation

$$
\begin{aligned}
& \underbrace{\left[\begin{array}{c}
\dot{\mathbf{s}} \\
\dot{\gamma}
\end{array}\right]}_{\dot{\mathbf{x}}}=\underbrace{\left[\begin{array}{cc}
\mathbf{A}_{\mathbf{s}} & \mathbf{B}_{\mathbf{s}} \mathbf{C}_{\boldsymbol{\gamma}} \\
\mathbf{0} & \mathbf{A}_{\boldsymbol{\gamma}}
\end{array}\right]}_{\mathbf{A}} \underbrace{\left[\begin{array}{c}
\mathbf{s} \\
\boldsymbol{\gamma}
\end{array}\right]}_{\mathbf{x}}+\underbrace{\left[\begin{array}{c}
\mathbf{0} \\
\mathbf{B}_{\gamma}
\end{array}\right]}_{\mathbf{B}_{w}} \mathbf{w}_{\boldsymbol{\gamma}} \\
& \underbrace{\left[\begin{array}{c}
\mathbf{y}_{s} \\
\mathbf{u}_{m}
\end{array}\right]}_{\mathbf{y}}=\underbrace{\left[\begin{array}{cc}
\mathbf{C}_{\mathbf{s}} & \mathbf{0} \\
\mathbf{0} & \mathbf{C}_{\gamma}
\end{array}\right]}_{\mathbf{C}} \mathbf{x}+\underbrace{\left[\begin{array}{c}
\mathbf{n}_{y} \\
\mathbf{n}_{u}
\end{array}\right]}_{\mathbf{n}}
\end{aligned}
$$

is also observable.

Proof. The observability matrix of the concatenated system $(\mathbf{C}, \mathbf{A})$ is

$$
\mathcal{O}=\left[\begin{array}{c}
\mathbf{C} \\
\mathbf{C A} \\
\vdots \\
\mathbf{C A}^{n_{s}+n_{\gamma}-1}
\end{array}\right]=\left[\begin{array}{cc}
\mathcal{O}_{\mathbf{s}} & \mathcal{O}_{12} \\
\mathbf{0} & \mathcal{O}_{\gamma} \\
\mathcal{O}_{31} & \mathcal{O}_{32}
\end{array}\right]
$$

where $n_{s}$ and $n_{\gamma}$ are respectively the dimension of the system $\left(\mathbf{C}_{\mathbf{s}}, \mathbf{A}_{\mathbf{s}}\right)$ and $\left(\mathbf{C}_{\gamma}, \mathbf{A}_{\gamma}\right), \mathcal{O}_{\mathbf{s}}$ and $\mathcal{O}_{\gamma}$ are the observability matrix of the two systems. Since $\left(\mathbf{C}_{\mathbf{s}}, \mathbf{A}_{\mathbf{s}}\right)$ and $\left(\mathbf{C}_{\gamma}, \mathbf{A}_{\gamma}\right)$ are both observable, $\mathcal{O}_{\mathrm{s}}$ and $\mathcal{O}_{\gamma}$ both has full column rank, which implies $\mathcal{O}$ has full column rank and the concatenated system $(\mathbf{C}, \mathbf{A})$ is observable.

\section{Case study}

Besides positing the dynamic behavior of a robotic system as a random process, we present another interpretation, from the perspective of signal processing, to our proposed motion model when it is combined with other techniques such as the Kalman filter. As proved in the previous section, the motion model, when concatenated to the kinematic model (3), remains observable, therefore a Kalman filter could be employed to estimate the full state vector $\mathbf{x}=\left[\begin{array}{ll}\mathbf{s}^{T} & \gamma^{T}\end{array}\right]^{T}$ and hence $\mathbf{u}=\mathbf{C}_{\boldsymbol{\gamma}} \boldsymbol{\gamma}$ from the measurements $\mathbf{u}_{m}$ and $\mathbf{y}$. Alternatively, the ground truth state $\mathbf{u}$ can be estimated by directly filtering $\mathbf{u}_{m}$. In this section, we present a simple case to illustrate the difference and connections between these two filters. In this simple case study, we assume that the dynamics state $u$ is the one-dimension linear acceleration $a$, the system kinematic model (3) is a velocity model, and the measurements are the velocity $v_{m}$ and acceleration $a_{m}$. We choose $N=2$ in the colored stochastic. The concatenated system is hence:

$$
\begin{aligned}
& \underbrace{\left[\begin{array}{c}
\dot{v} \\
\dot{a} \\
\dot{a}_{1}
\end{array}\right]}_{\dot{\mathbf{x}}}=\underbrace{\left[\begin{array}{ccc}
0 & 1 & 0 \\
0 & 0 & 1 \\
0 & 0 & 0
\end{array}\right]}_{\mathbf{A}} \underbrace{\left[\begin{array}{c}
v \\
a \\
a_{1}
\end{array}\right]}_{\mathbf{x}}+\underbrace{\left[\begin{array}{cc}
0 & 0 \\
1 & 0 \\
0 & 1
\end{array}\right]}_{\mathbf{B} w} \underbrace{\left[\begin{array}{c}
w_{a_{1}} \\
w_{a_{2}}
\end{array}\right]}_{\mathbf{w}} \\
& \underbrace{\left[\begin{array}{c}
v_{m} \\
a_{m}
\end{array}\right]}_{\mathbf{y}}=\underbrace{\left[\begin{array}{lll}
1 & 0 & 0 \\
0 & 1 & 0
\end{array}\right]}_{\mathbf{C}} \mathbf{x}+\underbrace{\left[\begin{array}{c}
n_{v} \\
n_{a}
\end{array}\right]}_{\mathbf{n}}
\end{aligned}
$$

where $w_{a_{1}} \sim \mathcal{N}\left(0, q_{a_{1}}\right), w_{a_{2}} \sim \mathcal{N}\left(0, q_{a_{2}}\right)$ are the process noises and $n_{v} \sim \mathcal{N}\left(0, r_{v}\right), n_{a} \sim \mathcal{N}\left(0, r_{a}\right)$ are the measurement noises.

As shown in the previous section, the concatenated system (5) is observable, hence the Kalman filter will converge to

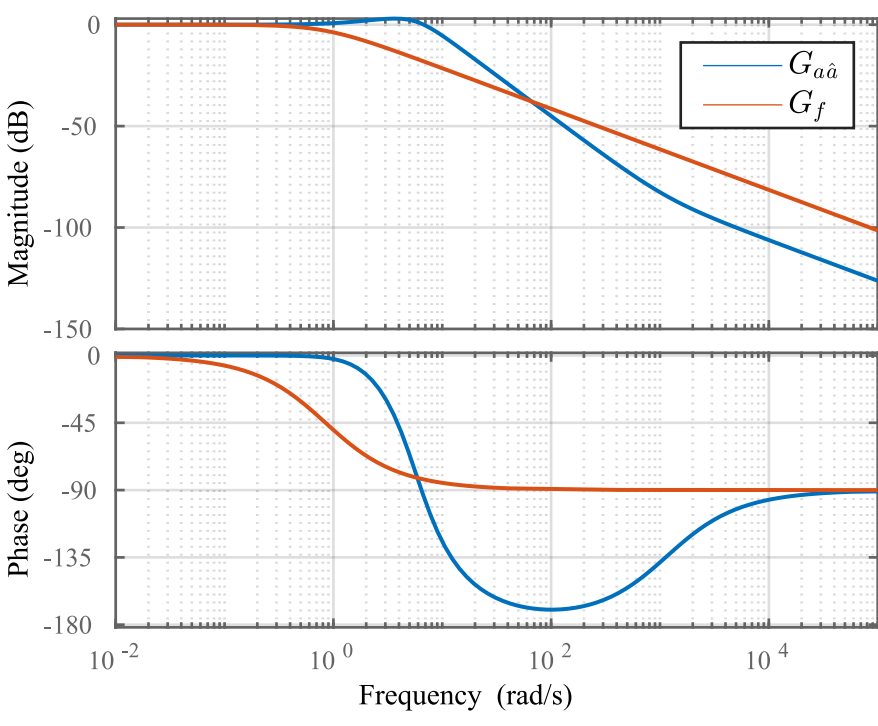

Fig. 4. The bode diagram of $G_{f}(s)$ and $G_{a \widehat{a}}(s)$ when $\left[\begin{array}{ll}r_{v} & r_{a}\end{array}\right]=$ $\left[\begin{array}{ll}0.1 & 1\end{array}\right]$ and $\left[\begin{array}{ll}q_{a_{1}} & q_{a_{2}}\end{array}\right]=\left[\begin{array}{ll}0.1 & 1\end{array}\right]$.

a stationary gain $\mathbf{L}_{\infty}$. At convergence, the transfer function from the combined output $\mathbf{y}$ to the acceleration estimation $\widehat{a}$ is

$$
\begin{aligned}
\widehat{a}(s) & =\mathbf{e}_{2}^{T}\left(s \mathbf{I}-\mathbf{A}+\mathbf{L}_{\infty} \mathbf{C}\right)^{-1} \mathbf{L}_{\infty} \mathbf{y}(s) \\
& =G_{v}(s) v_{m}(s)+G_{a}(s) a_{m}(s)
\end{aligned}
$$

where $\mathbf{e}_{2}=\left[\begin{array}{lll}0 & 1 & 0\end{array}\right]^{T}$ and $G_{v}(s), G_{a}(s)$ are the two components of $\mathbf{e}_{2}^{T}\left(s \mathbf{I}-\mathbf{A}+\mathbf{L}_{\infty} \mathbf{C}\right)^{-1} \mathbf{L}_{\infty}$.

Substituting the measurement model from (5) into (6) and recalling $v(s)=\frac{1}{s} a(s)$, we obtain

$\widehat{a}(s)=\left[\frac{G_{v}(s)}{s}+G_{a}(s)\right] a(s)+G_{v}(s) n_{v}(s)+G_{a}(s) n_{a}(s)$

Since the measurement noise $n_{v}$ and $n_{a}$ are independent, the standard deviation of $\widehat{a}$ caused by $n_{v}$ and $n_{a}$ is hence

$$
\sigma_{\widehat{a}}=\sqrt{r_{v}\left\|G_{v}(j \omega)\right\|_{2}^{2}+r_{a}\left\|G_{a}(j \omega)\right\|_{2}^{2}}
$$

And the transfer function from actual acceleration $a(s)$ to the estimated one $\widehat{a}(s)$ is:

$$
G_{a \widehat{a}}(s)=\frac{G_{v}(s)}{s}+G_{a}(s)
$$

Its bode diagram is shown in Fig. 4. It can be seen that $G_{a \widehat{a}}$ behaves like a first-order low-pass filter in high frequencies.

Moreover, if directly filtering the acceleration measurement $a_{m}$ by a first order Butterworth low-pass filter:

$$
G_{f}(s)=\frac{1}{1+k s}
$$

where the parameter $k$ is determined by ensuring the same level of noise attenuation (i.e., $\left\|G_{f}(j \omega)\right\|_{2}=$ $\left.\sqrt{r_{v}\left\|G_{v}(j \omega)\right\|_{2}^{2}+r_{a}\left\|G_{a}(j \omega)\right\|_{2}^{2}}\right)$. The transfer function $G_{f}(s)$ is shown in Fig. 4. It can be seen that the Kalman filter (i.e., $G_{a \widehat{a}}(s)$ ) has much higher bandwidth than the direct filtering method (i.e., $G_{f}(s)$ ), allowing more signal to pass through with low delay. This is no surprising as the Kalman 
filter infers this acceleration from both the acceleration and velocity measurements instead of one only. Finally, it is noticed that the Kalman filter has a slight amplification in the middle frequency range, which could lead to a small overshoot in the acceleration estimate.

\section{State Estimation}

In this section, we leverage the proposed motion model (1) to state estimations of robotic systems. We take an UAV shown in Fig. 5 as an illustrative example. This system is configured with an IMU sensor containing a gyroscope and an accelerometer and a position sensor (Pos in Fig.5) with orientation measurements, such as GPS, motion capture feedback, or visual-inertial odometry (VIO). The objective is to estimate the kinematics state (i.e., position, velocity, attitude, and IMU biases), dynamics states (i.e., angular velocity and linear acceleration), and the extrinsic parameters (i.e., the constant offset $\mathbf{c}$ between the position sensor and IMU).

\section{A. State Space Model}

To model the UAV system, we choose the IMU as the reference point and denote its position, specific acceleration, and body angular velocity as $\mathbf{p}$, a, and $\boldsymbol{\omega}$, respectively. It should be noted that the specific acceleration is the total acceleration removing the gravity and is directly measured by the accelerometer [55]. The rotation from inertial frame to IMU's body frame is denoted as a rotation matrix $\mathbf{R} \in S O(3)$. The measurement of position sensor, accelerometer, and gyroscope are $\mathbf{p}_{m}, \mathbf{a}_{m}$, and $\boldsymbol{\omega}_{m}$, respectively. The orientation measurement comes from magnetometer or other sensors like vision sensors and is denoted as $\mathbf{m}_{m}$. The biases of IMU accelerometer and gyroscope are denoted as $\mathbf{b}_{\mathbf{a}}$ and $\mathbf{b}_{\boldsymbol{\omega}}$, which are typically modeled as random walks [22]. With these notations, the resultant kinematic model is:

$$
\begin{aligned}
\dot{\mathbf{p}}=\mathbf{v}, & \dot{\mathbf{v}}=\mathbf{R} \mathbf{a}+\mathbf{g}, & \dot{\mathbf{b}}_{\mathbf{a}}=\mathbf{w}_{\mathbf{b}_{\mathbf{a}}} \\
\dot{\mathbf{c}}=\mathbf{0}, & \dot{\mathbf{R}}=\mathbf{R}\lfloor\boldsymbol{\omega}\rfloor, & \dot{\mathbf{b}}_{\boldsymbol{\omega}}=\mathbf{w}_{\mathbf{b}_{\boldsymbol{\omega}}}
\end{aligned}
$$

where the notation $\lfloor\cdot\rfloor$ denotes the skew-symmetric matrix of a vector $\cdot$ in $\mathbb{R}^{3}$. The system outputs are

$$
\begin{array}{ll}
\mathbf{a}_{m}=\mathbf{a}+\mathbf{b}_{\mathbf{a}}+\mathbf{n}_{\mathbf{a}}, & \boldsymbol{\omega}_{m}=\boldsymbol{\omega}+\mathbf{b}_{\boldsymbol{\omega}}+\mathbf{n}_{\boldsymbol{\omega}} \\
\mathbf{p}_{m}=\mathbf{p}+\mathbf{R} \mathbf{c}+\mathbf{n}_{\mathbf{p}}, & \mathbf{m}_{m}=\mathbf{R}^{T} \mathbf{e}_{1}+\mathbf{n}_{m}
\end{array}
$$

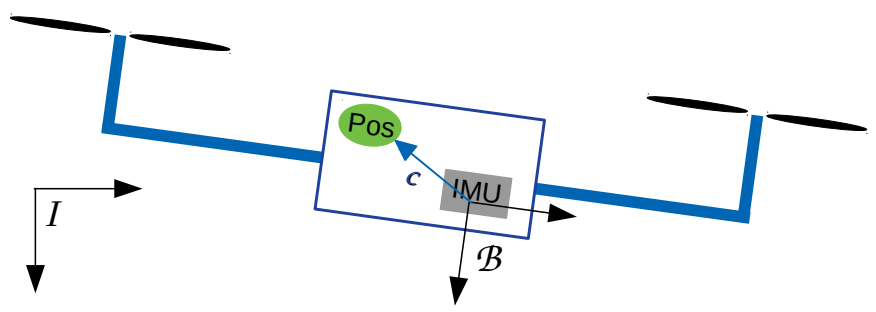

Fig. 5. A typical UAV configured with a position sensor and IMU.

\section{B. Input formulation}

The acceleration a and angular velocity $\boldsymbol{\omega}$ are unknown in (7). To form a valid state space model with known inputs, a common practice (e.g., [21, 25]-[28, 34, 35]) is to solve them from the measurements equation (8a), forming a system "driven" by the known measurement $\mathbf{a}_{m}$ and $\boldsymbol{\omega}_{m}$.

$$
\begin{aligned}
\dot{\mathbf{p}}=\mathbf{v}, & \dot{\mathbf{v}}=\mathbf{R}\left(\mathbf{a}_{m}-\mathbf{b}_{\mathbf{a}}-\mathbf{n}_{\mathbf{a}}\right)+\mathbf{g}, & \dot{\mathbf{b}}_{\mathbf{a}}=\mathbf{w}_{\mathbf{b}_{\mathbf{a}}}, \\
\dot{\mathbf{c}}=\mathbf{0}, & \dot{\mathbf{R}}=\mathbf{R}\left\lfloor\boldsymbol{\omega}_{m}-\mathbf{b}_{\boldsymbol{\omega}}-\mathbf{n}_{\boldsymbol{\omega}}\right\rfloor, & \dot{\mathbf{b}}_{\boldsymbol{\omega}}=\mathbf{w}_{\mathbf{b}_{\boldsymbol{\omega}}}
\end{aligned}
$$

We term this formulation as "input formulation" as the acceleration and angular velocity measurements appear as inputs to the formulated system. The system output vector is then $\mathbf{z}_{m}^{T}=\left[\begin{array}{ll}\mathbf{p}_{m}^{T} & \mathbf{m}_{m}^{T}\end{array}\right]$ in (8b). It should be noticed that a state estimator (e.g., EKF) based on the input formulation (9) does not filter the input signals $\mathbf{a}_{m}$ and $\boldsymbol{\omega}_{m}$.

\section{State formulation}

With the proposed statistical model (1), the unknown inputs in (7), a and $\boldsymbol{\omega}$, are now the outputs of (1), leading to a concatenated system shown below:

$$
\begin{array}{rlrl}
\dot{\gamma}_{\mathbf{a}} & =\mathbf{A}_{\gamma_{\mathbf{a}}} \gamma_{\mathbf{a}}+\mathbf{B}_{\gamma_{\mathbf{a}}} \mathbf{w}_{\boldsymbol{\gamma}_{\mathbf{a}}}, \dot{\gamma}_{\boldsymbol{\omega}}=\mathbf{A}_{\gamma_{\boldsymbol{\omega}}} \boldsymbol{\gamma}_{\boldsymbol{\omega}}+\mathbf{B}_{\gamma_{\boldsymbol{\omega}}} \mathbf{w}_{\gamma_{\boldsymbol{\omega}}} \\
\dot{\mathbf{p}} & =\mathbf{v}, \quad \dot{\mathbf{v}}=\mathbf{R}\left(\mathbf{C}_{\gamma_{\mathbf{a}}} \boldsymbol{\gamma}_{\mathbf{a}}\right)+\mathbf{g}, & \dot{\mathbf{b}}_{\mathbf{a}}=\mathbf{w}_{\mathbf{b}_{\mathbf{a}}}, \\
\dot{\mathbf{c}} & =\mathbf{0}, & \dot{\mathbf{R}}=\mathbf{R}\left\lfloor\mathbf{C}_{\gamma_{\boldsymbol{\omega}}} \boldsymbol{\gamma}_{\boldsymbol{\omega}}\right\rfloor, & \dot{\mathbf{b}}_{\boldsymbol{\omega}}=\mathbf{w}_{\mathbf{b}_{\boldsymbol{\omega}}}
\end{array}
$$

The system output vector is $\mathbf{z}_{m}^{T}=\left[\begin{array}{llll}\mathbf{p}_{m}^{T} & \mathbf{m}_{m}^{T} & \mathbf{a}_{m}^{T} & \boldsymbol{\omega}_{m}^{T}\end{array}\right]$ as in (8). Unlike the input formulation (9), the dynamics states $\mathbf{a}=\mathbf{C}_{\gamma_{\mathbf{a}}} \gamma_{\mathbf{a}}$ and $\boldsymbol{\omega}=\mathbf{C}_{\boldsymbol{\gamma}_{\boldsymbol{\omega}}} \boldsymbol{\gamma}_{\boldsymbol{\omega}}$ now appear as functions of system states, thus could be filtered by a state estimator. We term this new formulation as "state formulation".

\section{Estimation}

To estimate the dynamics states a and $\boldsymbol{\omega}$, it is sufficient to estimate the state vector of the state formulation (10). This is a standard state estimation problem and various nonlinear state estimator could be used such as EKF and UKF. In this paper, we employ an error-state EKF as detailed in [21]. The filter error-state vector is defined as

$$
\widetilde{\mathbf{x}}=\left[\begin{array}{llllllll}
\widetilde{\mathbf{p}}^{T} & \widetilde{\mathbf{v}}^{T} & \delta \boldsymbol{\theta}^{T} & \widetilde{\mathbf{c}}^{T} & \widetilde{\mathbf{b}}_{a}^{T} & \widetilde{\mathbf{b}}_{\omega}^{T} & \widetilde{\boldsymbol{\gamma}}_{\mathbf{a}}^{T} & \widetilde{\boldsymbol{\gamma}}_{\boldsymbol{\omega}}^{T}
\end{array}\right]^{T}
$$

where $\delta \boldsymbol{\theta}=\log \left(\widehat{\mathbf{R}}^{T} \mathbf{R}\right)$ is the attitude error and the rests are standard additive errors (i.e., the error in the estimate $\widehat{\mathbf{x}}$ of a quantity $\mathbf{x}$ is $\widetilde{\mathbf{x}}=\mathbf{x}-\widehat{\mathbf{x}}$ ). Intuitively, the attitude error $\delta \boldsymbol{\theta}$ describes the (small) deviation between the true and the estimated attitude. The main advantage of this error definition is that it allows us to represent the attitude uncertainty by the $3 \times 3$ covariance matrix $\mathbb{E}\left\{\delta \boldsymbol{\theta} \delta \boldsymbol{\theta}^{T}\right\}$. Since the attitude has 3 degree of freedom (DOF), this is a minimal representation.

\section{1) Filter Propagation:}

The propagation step breaks into state propagation and covariance propagation. The former one is achieved by applying expectation operator on both side of (10). The propagation on covariance is applied on the error state vector (11) by linearizing the state equation (10):

$$
\dot{\tilde{\mathbf{x}}}=\mathbf{F}_{\mathbf{x}} \widetilde{\mathbf{x}}+\mathbf{F}_{\mathbf{w}} \mathbf{w}
$$


where $\mathbf{F}_{\mathbf{x}}, \mathbf{F}_{\mathbf{w}}$ are the Jacobin matrices w.r.t. $\widetilde{\mathbf{x}}$, and $\mathbf{w} \sim$ $\mathcal{N}(\mathbf{0}, \mathcal{Q})$ is the process noise.

The covariance matrix is then propagated as

$$
\mathbf{P}_{k+1 \mid k}=\boldsymbol{\Phi}_{\mathbf{x}_{k}} \mathbf{P}_{k \mid k} \boldsymbol{\Phi}_{\mathbf{x}_{k}}^{T}+\boldsymbol{\Phi}_{\mathbf{w}_{k}} \mathcal{Q} \boldsymbol{\Phi}_{\mathbf{w}_{k}}^{T}
$$

where $\boldsymbol{\Phi}_{\mathbf{x}_{k}}=\mathbf{F}_{\mathbf{x}_{k}} \cdot \Delta t+\mathbf{I}$ and $\boldsymbol{\Phi}_{\mathbf{w}_{k}}=\mathbf{F}_{\mathbf{w}_{k}} \cdot \Delta t$.

2) Filter Update:

The measurement residual is

$$
\mathbf{r}=\mathbf{z}_{m}-\hat{\mathbf{z}} \simeq \mathbf{H} \widetilde{\mathbf{x}}+\mathbf{n}
$$

where $\mathbf{H}$ is the Jacobin matrices of (8) w.r.t. $\widetilde{\mathbf{x}}$, and $\mathbf{n} \sim$ $\mathcal{N}(\mathbf{0}, \mathcal{R})$ is the measurement noise vector in (8). Every time there comes a new measurement, the state variables are updated through the following steps:

a). Compute the Kalman gain:

$$
\mathbf{K}_{k+1}=\mathbf{P}_{k+1 \mid k} \mathbf{H}_{k+1}^{T}\left(\mathbf{H}_{k+1} \mathbf{P}_{k+1 \mid k} \mathbf{H}_{k+1}^{T}+\mathcal{R}\right)^{-1}
$$

b). Update the Error states:

$$
\widetilde{\mathbf{x}}_{k+1 \mid k+1}=\mathbf{K}_{k+1}\left(\mathbf{z}_{m_{k+1}}-\hat{\mathbf{z}}_{k+1}\right)
$$

c). Update the original state variables based on the definition of the error state in (11), and compute the covariance as:

$$
\mathbf{P}_{k+1 \mid k+1}=\mathbf{P}_{k+1 \mid k}-\mathbf{K}_{k+1} \mathbf{S}_{k+1} \mathbf{K}_{k+1}^{T}
$$

\section{OBSERVABILITy ANALYSIS}

For a filter (e.g., EKF) to converge, the system must be observable [45]. For linear time invariant (LTI) systems, the observability is easily determined by the well-known linear observability matrix rank test [56]. For nonlinear systems, the observability is much more complicated [46]. Among the various variants of observability outlined in [46], the locally weakly observability has been widely used in robotics for state estimation [48]-[50, 57], online extrinsic calibration [34, 35], and parameter identification $[33,51]$, etc.

A system is said to be locally weakly observable if its state can be distinguished instantaneously (i.e., requiring arbitrarily small time duration) from any other states in a certain neighbor. Intuitively, this means a state estimator (e.g., EKF) will converge to an unbiased estimate of the state if the model truthfully represents the reality and the system inputs have sufficient excitation. An advantage of the locally weakly observability is that it lends itself to a simple algebraic test known as the observability rank condition [46].

\section{A. Observability Rank Condition} $\mathcal{M}$ :

Consider an input-linear system [58] on smooth manifold

$$
\begin{aligned}
& \dot{\mathbf{x}}=\mathbf{f}_{0}(\mathbf{x})+\sum_{i=1}^{m} \mathbf{f}_{i}(\mathbf{x}) u_{i} \\
& \mathbf{y}=\mathbf{h}(\mathbf{x})
\end{aligned}
$$

where $\mathbf{x} \in \mathcal{M}, \mathbf{f}_{0}(\mathbf{x})$ is the drift (i.e., zero-input) vector field, and $\mathbf{f}_{i}(\mathbf{x}), i=1, \cdots, m$, defines a vector field on $\mathcal{M}$ that is linear to each control input. Then the observability rank condition implies that if the observability matrix $\mathcal{O}(\overline{\mathbf{x}})$ defined below has full column rank at $\overline{\mathbf{x}}$, system (12) is locally weakly observable at $\overline{\mathbf{x}}$ [46]. Furthermore, if $\mathcal{O}(\overline{\mathbf{x}})$ has full column rank for all $\overline{\mathrm{x}} \in \mathcal{M}$, then the system is locally weakly observable [59].

$$
\mathcal{O}(\overline{\mathbf{x}})=\left[\begin{array}{c}
\vdots \\
\nabla_{\mathbf{x}}\left(\left(\mathcal{L}_{\mathbf{f}^{k} \ldots \mathbf{f}^{1}}^{k} \mathbf{h}\right)(\mathbf{x})\right)(\overline{\mathbf{x}}) \\
\vdots
\end{array}\right]
$$

where $k=0,1, \ldots$, and $\mathbf{f}^{k}$ is any of $\left\{\mathbf{f}_{0}, \mathbf{f}_{1}, \ldots, \mathbf{f}_{m}\right\}$. When $k=1,\left(\mathcal{L}_{\mathbf{f}}^{1} \mathbf{h}\right)(\mathbf{x})$ is the Lie derivative of function $\mathbf{h}$ along the vector field $\mathrm{f}$ at point $\mathrm{x} \in \mathcal{M}$ and is defined below [60]

$$
\begin{aligned}
\left(\mathcal{L}_{\mathbf{f}}^{1} \mathbf{h}\right)(\mathbf{x}) & =\left.\frac{d \mathbf{h}(\mathbf{r}(t))}{d t}\right|_{t=0}, \\
& \text { subject to } \frac{d \mathbf{r}(t)}{d t}=\mathbf{f}(\mathbf{r}(t)), \mathbf{r}(0)=\mathbf{x}
\end{aligned}
$$

Since the time derivative of $\mathbf{h}(\mathbf{x}(t))$ is evaluated at time zero, it is sufficient to only consider $\mathbf{r}(t)$ at $t=0$, i.e., $\dot{\mathbf{r}}(0)=$ $\mathbf{f}(\mathbf{r}(0)), \mathbf{r}(0)=\mathbf{x}$. Then (14) is equivalent to the following form for the sake of notation simplicity

$$
\left(\mathcal{L}_{\mathbf{f}}^{1} \mathbf{h}\right)(\mathbf{x})=\dot{\mathbf{h}}(\mathbf{x}), \text { subject to } \dot{\mathbf{x}}=\mathbf{f}(\mathbf{x})
$$

In particular, when $\mathcal{M}=\mathbb{R}^{n}$, the Lie derivative in (15) can be simplified by the chain rule and becomes the commonly seen form.

$$
\left(\mathcal{L}_{\mathbf{f}}^{1} \mathbf{h}\right)(\mathbf{x})=\frac{\partial \mathbf{h}(\mathbf{x})}{\partial \mathbf{x}} \mathbf{f}(\mathbf{x})
$$

Based on the first order Lie derivative defined in (15), the higher order Lie derivatives $\left(\mathcal{L}_{\mathbf{f}^{k} \ldots \mathbf{f}^{1}}^{k} \mathbf{h}\right)(\mathbf{x})$ in (13) is recursively defined as follows.

$$
\begin{aligned}
\left(\mathcal{L}^{0} \mathbf{h}\right)(\mathbf{x}) & =\mathbf{h}(\mathbf{x}) \\
\left(\mathcal{L}_{\mathbf{f}^{k} \ldots \mathbf{f}^{1}}^{k} \mathbf{h}\right)(\mathbf{x}) & =\left(\mathcal{L}_{\mathbf{f}^{k}}\left(\mathcal{L}_{\mathbf{f}^{k-1} \ldots \mathbf{f}^{1}}^{k-1} \mathbf{h}\right)\right)(\mathbf{x})
\end{aligned}
$$

As can be seen, $\left(\mathcal{L}_{\mathbf{f}^{k} \ldots \mathbf{f}^{1}}^{k} \mathbf{h}\right)(\mathbf{x})$ is a function of $\mathbf{x} \in \mathcal{M}$. To simplify the notation, we denote it as $\mathbf{g}(\mathbf{x})$. Then, the notation $\nabla_{\mathbf{x}}(\mathbf{g}(\mathbf{x}))(\overline{\mathbf{x}})$ in (13) denotes the gradient of $\mathbf{g}(\mathbf{x}): \mathcal{M} \mapsto$ $\mathbb{R}^{m}$ w.r.t. $\mathbf{x}$ evaluated at $\overline{\mathbf{x}}$. If $\mathcal{M}=\mathbb{R}^{n}$, this becomes the well known partial derivation.

$$
\nabla_{\mathbf{x}}(\mathbf{g}(\mathbf{x}))(\overline{\mathbf{x}})=\left.\frac{\partial \mathbf{g}(\mathbf{x})}{\partial \mathbf{x}}\right|_{\mathbf{x}=\overline{\mathbf{x}}} \in \mathbb{R}^{m \times n}
$$

For a generic smooth manifold $\mathcal{M}$ of dimension $n$, a local coordinate chart around $\overline{\mathbf{x}}$ on $\mathcal{M}$ needs to be found (see Fig. 6). Assume the local coordinate chart is $(U, \varphi)$, where $U$ is an open subset of $\mathcal{M}$ containing the point $\overline{\mathrm{x}}$ and $\varphi: U \mapsto \widehat{U}$ is a homeomorphism from $U$ to an open subset $\widehat{U}=\varphi(U) \subset \mathbb{R}^{n}$. Then the gradient of $\mathbf{g}(\mathbf{x})$ is computed as [61]:

$$
\nabla_{\mathbf{x}}(\mathbf{g}(\mathbf{x}))(\overline{\mathbf{x}})=\left.\frac{\partial \mathbf{g}\left(\boldsymbol{\varphi}^{-1}(\boldsymbol{\theta})\right)}{\partial \boldsymbol{\theta}}\right|_{\boldsymbol{\theta}=\boldsymbol{\varphi}(\overline{\mathbf{x}})}
$$

In particular, if $\mathcal{M}=S O(3)$, one such local coordinate chart is $(U, \varphi)$, where $U=\left\{\mathbf{x} \in S O(3) \mid\left\|\log \left(\overline{\mathbf{x}}^{-1} \mathbf{x}\right)\right\|_{2}<\pi\right\}$ and $\varphi(\mathbf{x})=\log \left(\overline{\mathbf{x}}^{-1} \mathbf{x}\right) \in \mathbb{R}^{3}$. "Log" is the logarithmic function that maps a rotation matrix to its rotation vector $[62,63]$ 


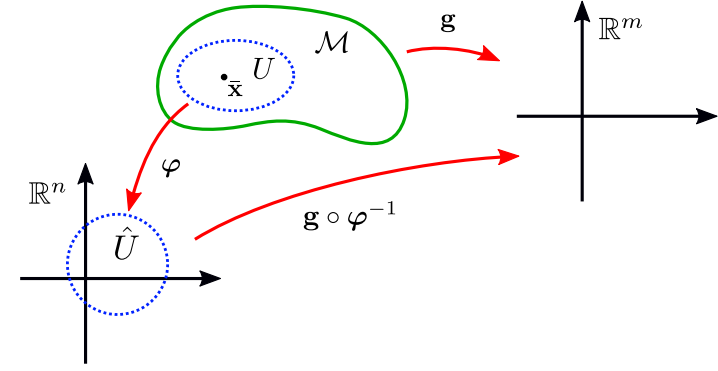

Fig. 6. Illustration of a coordinate chart $(U, \boldsymbol{\varphi})$ on manifold $\mathcal{M}$.

and its inverse map is denoted as "Exp". As a result, the gradient of $\mathbf{g}(\mathbf{x}): S O(3) \mapsto \mathbb{R}^{m}$ is

$$
\nabla_{\mathbf{x}}(\mathbf{g}(\mathbf{x}))(\overline{\mathbf{x}})=\left.\frac{\partial \mathbf{g}(\overline{\mathbf{x}} \cdot \operatorname{Exp}(\boldsymbol{\theta}))}{\partial \boldsymbol{\theta}}\right|_{\boldsymbol{\theta}=\mathbf{0}}
$$

It should be noted that the coordinate charts are not unique and a variety of alternatives could also be used. Two apparent alternatives are 1) using different (minimal) rotation parameterizations (e.g., Euler angles) instead of rotation vector as the local coordinates $\theta$; and 2) off-centering the map $\varphi(\mathbf{x})$ from $\overline{\mathbf{x}}$. The first alternative will lead to the same partial differentiation as (20) while the second one produces an additional Jacobian matrix. Nevertheless, the rank of the observability matrix (13) are identical in all cases, hence different coordinate chart does not affect the system observability. In fact, the "Exp/Log" map used in (20) lends itself a simple observability matrix for rank analysis, as will be seen in the following subsections. This parameterization is also widely used in $S O(3)$ optimizations [64]-[66].

\section{B. Observability of Input formulation}

In this section, we analyze the observability of the input formulation (9) based on the observability rank condition outlined above. Similar (or even more complicated) observability analysis have been explored before in visual-inertial navigation [49], camera-IMU extrinsic calibration [34, 35], and parameter identification [33]. In all these work, the quaternion $\mathbf{q}$ is used to represent robots' attitude and is treated as a flat vector in $\mathbb{R}^{4}$ to compute the Lie derivatives as in (16) and partial derivations as in (18). This method suffers from several drawbacks: 1) In order to compensate for the over-parameterization when using quaternion, an additional virtual measurement $\mathbf{q}^{T} \mathbf{q}=1$ must be used to prevent rank deficiency; 2) The over-parameterization in state vector and additional virtual measurement lead to a rather complicated observability matrix, the resultant rank analysis is also rather difficult. Applying such analysis to higher order systems (e.g., state formulation (10)) is cumbersome; 3) Viewing quaternion $\mathbf{q}$ as a vector in $\mathbb{R}^{4}$ undermines the structure of the unit quaternion. More concretely, the locally weakly observability based on $\mathbf{q} \in \mathbb{R}^{4}$ implies a neighborhood where the distance is defined as $d\left(\mathbf{q}_{1}, \mathbf{q}_{2}\right)=\left\|\mathbf{q}_{1}-\mathbf{q}_{2}\right\|_{2}$, which is not a natural metric for $S O(3)$ (i.e., it does not form $S O(3)$ into a Riemannian manifold).
We show that all these drawbacks can be avoided if we develop the Lie derivatives directly on manifold as in (15) and (17) and the associated partial derivations as in (19). The resultant observaiblity analysis is 1) Simple, it does not need to over-parameterize the state vector hence requiring no virtual measurement. The resultant observability matrix is easy for rank analysis; 2) Scalable, the simple and compact observability matrix allows the analysis to be conducted for more complicated and higher order systems such as the state formulation (10); and 3) Natural, since the observability analysis is conducted directly on manifold, the neighborhood it implies is based on the natural metric that forms $\mathcal{M}$ into a Riemannian manifold. For $S O(3)$, the metric is $d\left(\mathbf{R}_{1}, \mathbf{R}_{2}\right)=$ $\left\|\log \left(\mathbf{R}_{2}^{T} \mathbf{R}_{1}\right)\right\|_{2}$, which is a geodesic on $S O(3)$ [67].

To analyze the observability of the input formulation (9), we ignore the noise $\mathbf{w}$ and $\mathbf{n}$ and rewrite it as an input-linear system as below:

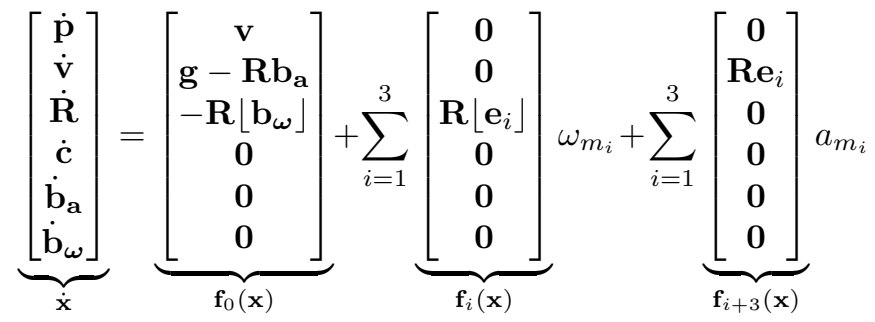

$\mathbf{h}_{1}(\mathbf{x})=\mathbf{p}+\mathbf{R c}, \quad \mathbf{h}_{2}(\mathbf{x})=\mathbf{R}^{T} \mathbf{e}_{1}$

1) Zero-order Lie derivatives $\left(\mathcal{L}^{0} \mathbf{h}_{1}, \mathcal{L}^{0} \mathbf{h}_{2}\right)$ : By the definition in $(17 \mathrm{a})$, we have

$$
\begin{aligned}
& \left(\mathcal{L}^{0} \mathbf{h}_{1}\right)(\mathbf{x})=\mathbf{p}+\mathbf{R c} \\
& \left(\mathcal{L}^{0} \mathbf{h}_{2}\right)(\mathbf{x})=\mathbf{R}^{T} \mathbf{e}_{1}
\end{aligned}
$$

and the partial derivation based on (20)

$$
\begin{aligned}
& \nabla_{\mathbf{x}}\left(\left(\mathcal{L}^{0} \mathbf{h}_{1}\right)(\mathbf{x})\right)(\overline{\mathbf{x}})=\left[\begin{array}{llllll}
\mathbf{I} & \mathbf{0} & -\overline{\mathbf{R}}\lfloor\overline{\mathbf{c}}\rfloor & \overline{\mathbf{R}} & \mathbf{0} & \mathbf{0}
\end{array}\right] \\
& \nabla_{\mathbf{x}}\left(\left(\mathcal{L}^{0} \mathbf{h}_{2}\right)(\mathbf{x})\right)(\overline{\mathbf{x}})=\left[\begin{array}{llllll}
\mathbf{0} & \mathbf{0} & \left\lfloor\overline{\mathbf{R}}^{T} \mathbf{e}_{1}\right\rfloor & \mathbf{0} & \mathbf{0} & \mathbf{0}
\end{array}\right]
\end{aligned}
$$

2) First-order Lie derivatives $\left(\mathcal{L}_{\mathbf{f}_{0}}^{1} \mathbf{h}_{1}, \mathcal{L}_{\mathbf{f}_{1}}^{1} \mathbf{h}_{1}, \mathcal{L}_{\mathbf{f}_{2}}^{1} \mathbf{h}_{1}, \mathcal{L}_{\mathbf{f}_{0}}^{1} \mathbf{h}_{2}\right)$ : From (14), we have

$$
\begin{aligned}
& \left(\mathcal{L}_{\mathbf{f}_{0}}^{1} \mathbf{h}_{1}\right)(\mathbf{x})=\dot{\mathbf{h}}_{1}(\mathbf{x}), \quad \text { subject to } \dot{\mathbf{x}}=\mathbf{f}_{0}(\mathbf{x}) \\
& =\mathbf{v}+\dot{\mathbf{R}} \mathbf{c}, \quad \text { subject to } \dot{\mathbf{R}}=-\mathbf{R}\left\lfloor\mathbf{b}_{\boldsymbol{\omega}}\right\rfloor \\
& =\mathbf{v}-\mathbf{R}\left\lfloor\mathbf{b}_{\boldsymbol{\omega}}\right\rfloor \mathbf{c}
\end{aligned}
$$

Hence, the first order Lie derivatives are

$$
\begin{aligned}
& \left(\mathcal{L}_{\mathbf{f}_{0}}^{1} \mathbf{h}_{1}\right)(\mathbf{x})=\mathbf{v}-\mathbf{R}\left\lfloor\mathbf{b}_{\boldsymbol{\omega}}\right\rfloor \mathbf{c} \\
& \left(\mathcal{L}_{\mathbf{f}_{i}}^{1} \mathbf{h}_{1}\right)(\mathbf{x})=\mathbf{R}\left\lfloor\mathbf{e}_{i}\right\rfloor \mathbf{c}, i=1,2,3 \\
& \left(\mathcal{L}_{\mathbf{f}_{0}}^{1} \mathbf{h}_{2}\right)(\mathbf{x})=\left\lfloor\mathbf{b}_{\boldsymbol{\omega}}\right\rfloor \mathbf{R}^{T} \mathbf{e}_{1}
\end{aligned}
$$

and the associated partial derivations are

$$
\begin{aligned}
& \nabla_{\mathbf{x}}\left(\left(\mathcal{L}_{\mathbf{f}_{0}}^{1} \mathbf{h}_{1}\right)(\mathbf{x})\right)(\overline{\mathbf{x}})=\left[\begin{array}{llllll}
\mathbf{0} & \mathbf{I} & \bullet & \bullet & \mathbf{0} & \bullet
\end{array}\right] \\
& \nabla_{\mathbf{x}}\left(\left(\mathcal{L}_{\mathbf{f}_{i}}^{1} \mathbf{h}_{1}\right)(\mathbf{x})\right)(\overline{\mathbf{x}})=\left[\begin{array}{llllll}
\mathbf{0} & \mathbf{0} & \bullet & \overline{\mathbf{R}}\left\lfloor\mathbf{e}_{i}\right.
\end{array}\right] \\
& \nabla_{\mathbf{x}}\left(\left(\mathcal{L}_{\mathbf{f}_{0}}^{1} \mathbf{h}_{2}\right)(\mathbf{x})\right)(\overline{\mathbf{x}})=\left[\begin{array}{llllll}
\mathbf{0} & \mathbf{0} & \bullet & \mathbf{0} & \mathbf{0} & -\lfloor\overline{\boldsymbol{\beta}}\rfloor
\end{array}\right]
\end{aligned}
$$

where $\overline{\boldsymbol{\beta}}=\overline{\mathbf{R}}^{T} \mathbf{e}_{1}$ and those $\bullet$ are elements that do not affect the observability analysis but can be calculated easily when required. 
3) Second-order Lie derivatives $\left(\mathcal{L}_{\mathbf{f}_{0} \mathbf{f}_{0}}^{2} \mathbf{h}_{1}, \quad \mathcal{L}_{\mathbf{f}_{i+3} \mathbf{f}_{0}}^{2} \mathbf{h}_{1}\right.$, $\left.\mathcal{L}_{\mathbf{f}_{i} \mathbf{f}_{0}}^{2} \mathbf{h}_{2}, i=1,2,3\right)$ : Finally, we calculate the second order Lie derivatives based on the recursive definition in (17b):

$$
\begin{aligned}
\left(\mathcal{L}_{\mathbf{f}_{0} \mathbf{f}_{0}}^{2} \mathbf{h}_{1}\right)(\mathbf{x}) & =\mathcal{L}_{\mathbf{f}_{0}}^{1}\left(\mathcal{L}_{\mathbf{f}_{0}}^{1} \mathbf{h}_{1}\right)(\mathbf{x})=\frac{d\left(\left(\mathcal{L}_{\mathbf{f}_{0}}^{1} \mathbf{h}_{1}\right)(\mathbf{x})\right)}{d t} \\
& =\dot{\mathbf{v}}-\dot{\mathbf{R}}\left\lfloor\mathbf{b}_{\boldsymbol{\omega}}\right\rfloor \mathbf{c}, \quad \text { subject to } \dot{\mathbf{x}}=\mathbf{f}_{0}(\mathbf{x}) \\
& =\mathbf{g}-\mathbf{R} \mathbf{b}_{\mathbf{a}}+\mathbf{R}\left\lfloor\mathbf{b}_{\boldsymbol{\omega}}\right\rfloor^{2} \mathbf{c}
\end{aligned}
$$

Hence,

$$
\begin{aligned}
\left(\mathcal{L}_{\mathbf{f}_{0} \mathbf{f}_{0}}^{2} \mathbf{h}_{1}\right)(\mathbf{x}) & =\mathbf{g}-\mathbf{R} \mathbf{b}_{\mathbf{a}}+\mathbf{R}\left\lfloor\mathbf{b}_{\boldsymbol{\omega}}\right\rfloor^{2} \mathbf{c} \\
\left(\mathcal{L}_{\mathbf{f}_{i+3} \mathbf{f}_{0}}^{2} \mathbf{h}_{1}\right)(\mathbf{x}) & =\mathbf{R e}_{i}, i=1,2,3 \\
\left(\mathcal{L}_{\mathbf{f}_{i} \mathbf{f}_{0}}^{2} \mathbf{h}_{2}\right)(\mathbf{x}) & =\left\lfloor\mathbf{b}_{\boldsymbol{\omega}}\right\rfloor\left\lfloor\mathbf{R}^{T} \mathbf{e}_{1}\right\rfloor \mathbf{e}_{i}, i=1,2,3
\end{aligned}
$$

and the associated partial derivations can be obtained.

Putting together all the partial derivations and replacing $\overline{\mathbf{x}}$ with $\mathbf{x}$ for the sake of notation simplicity, we obtain the following observability matrix:

$$
\mathcal{O}_{I}=\left[\begin{array}{c}
\nabla_{\mathbf{x}} \mathcal{L}^{0} \mathbf{h}_{1} \\
\nabla_{\mathbf{x}} \mathcal{L}_{\mathbf{f}_{0}}^{1} \mathbf{h}_{1} \\
\nabla_{\mathbf{x}} \mathcal{L}_{\mathbf{f}_{\mathbf{f}}}^{1} \mathbf{h}_{1} \\
\nabla_{\mathbf{x}} \mathcal{L}_{\mathbf{f}_{2}}^{1} \mathbf{h}_{1} \\
\nabla_{\mathbf{x}} \mathcal{L}_{\mathbf{f}_{0}}^{2} \mathbf{h}_{0} \mathbf{h}_{1} \\
\nabla_{\mathbf{x}} \mathcal{L}_{\mathbf{f}_{\mathbf{f}} \mathbf{f}_{0}}^{2} \mathbf{h}_{1} \\
\nabla_{\mathbf{x}} \mathcal{L}_{\mathbf{f}_{\mathbf{f}}}^{2} \mathbf{h}_{1} \\
\nabla_{\mathbf{x}} \mathcal{L}_{\mathbf{f}_{0}}^{1} \mathbf{h}_{2} \\
\nabla_{\mathbf{x}} \mathcal{L}_{\mathbf{f}_{1}}^{2} \mathbf{f}_{\mathbf{f}_{0}} \mathbf{h}_{2} \\
\nabla_{\mathbf{x}} \mathcal{L}_{\mathbf{f}_{2} \mathbf{f}_{0}}^{2} \mathbf{h}_{2}
\end{array}\right]=\left[\begin{array}{cccccc}
\mathbf{I} & \mathbf{0} & \bullet & \bullet & \mathbf{0} & \mathbf{0} \\
\mathbf{0} & \mathbf{I} & \bullet & \bullet & \mathbf{0} & \bullet \\
\mathbf{0} & \mathbf{0} & \bullet & \mathbf{R}\left\lfloor\mathbf{e}_{1}\right\rfloor & \mathbf{0} & \mathbf{0} \\
\mathbf{0} & \mathbf{0} & \bullet & \mathbf{R}\left\lfloor\mathbf{e}_{2}\right\rfloor & \mathbf{0} & \mathbf{0} \\
\mathbf{0} & \mathbf{0} & \bullet & \dot{\bullet} & -\mathbf{R} & \bullet \\
\mathbf{0} & \mathbf{0} & -\mathbf{R}\left\lfloor\mathbf{e}_{1}\right\rfloor & \mathbf{0} & \mathbf{0} & \mathbf{0} \\
\mathbf{0} & \mathbf{0} & -\mathbf{R}\left\lfloor\mathbf{e}_{2}\right\rfloor & \mathbf{0} & \mathbf{0} & \mathbf{0} \\
\mathbf{0} & \mathbf{0} & \bullet & \mathbf{0} & \mathbf{0} & -\lfloor\boldsymbol{\beta}\rfloor \\
\mathbf{0} & \mathbf{0} & \bullet & \mathbf{0} & \mathbf{0} & -\left\lfloor\lfloor\boldsymbol{\beta}\rfloor \mathbf{e}_{1}\right\rfloor \\
\mathbf{0} & \mathbf{0} & \bullet & \mathbf{0} & \mathbf{0} & -\left\lfloor\lfloor\boldsymbol{\beta}\rfloor \mathbf{e}_{2}\right\rfloor
\end{array}\right]
$$

which apparently has full column rank since $\left\lfloor\mathbf{e}_{1}\right\rfloor \mathbf{v}=\mathbf{0}$ and $\left\lfloor\mathbf{e}_{2}\right\rfloor \mathbf{v}=\mathbf{0}$ cannot hold simultaneously for any nonzero vector $\mathbf{v}$. Therefore, the input formulation (9) is observable. Moreover, It is obviously that when compared with the observability analysis using quaternion parameterization [34, 35], the observability matrix $\mathcal{O}_{I}$ is much simpler and its rank is immediately available.

\section{Observability of State formulation}

In this section, we show that the observability analysis previously developed on manifold is scalable to higher order systems such as the state formulation (10). The system can be rewritten as a more compact form as below

$$
\begin{aligned}
& \dot{\mathbf{x}}=\mathbf{f}_{0}(\mathbf{x}) \\
& \mathbf{h}_{1}(\mathbf{x})=\mathbf{p}+\mathbf{R} \mathbf{c}, \mathbf{h}_{3}(\mathbf{x})=\mathbf{b}_{\mathbf{a}}+\mathbf{C}_{\boldsymbol{\gamma}_{\mathbf{a}}} \gamma_{\mathbf{a}} \\
& \mathbf{h}_{2}(\mathbf{x})=\mathbf{R}^{T} \mathbf{e}_{1}, \quad \mathbf{h}_{4}(\mathbf{x})=\mathbf{b}_{\boldsymbol{\omega}}+\mathbf{C}_{\gamma_{\boldsymbol{\omega}}} \gamma_{\boldsymbol{\omega}}
\end{aligned}
$$

where the function $\mathbf{f}_{0}(\mathbf{x})$ comes from (10) when ignoring all the white noises. This system has no exogenous inputs, so only Lie derivatives $\mathcal{L}_{\mathrm{f}_{0} \ldots \mathrm{f}_{0}}^{k}$ are required in determining the system observability. Further denoting

$$
{ }_{k}^{i} \mathcal{G}_{\mathbf{x}}^{\mathbf{h}}(\overline{\mathbf{x}})=\left[\begin{array}{c}
\nabla_{\mathbf{x}}\left(\left(\mathcal{L}_{\mathbf{f}_{0} \cdots \mathbf{f}_{0}}^{i} \mathbf{h}\right)(\mathbf{x})\right)(\overline{\mathbf{x}}) \\
\vdots \\
\nabla_{\mathbf{x}}\left(\left(\mathcal{L}_{\mathbf{f}_{0} \cdots \mathbf{f}_{0}}^{k} \mathbf{h}\right)(\mathbf{x})\right)(\overline{\mathbf{x}})
\end{array}\right]
$$

and denoting $\overline{\mathbf{x}}$ as $\mathbf{x}$, we obtain the observability matrix $\mathcal{O}_{S}$ shown in (24), where $\mathcal{O}_{\gamma_{\mathrm{a}}}$ and $\mathcal{O}_{\gamma_{\mathrm{a}}}$ is the observability matrix of system $\left(\mathbf{C}_{\gamma_{\mathrm{a}}}, \mathbf{A}_{\gamma_{\mathrm{a}}}, \mathbf{B}_{\gamma_{\mathrm{a}}}\right)$ of dimension $n_{a}$ and $\left(\mathbf{C}_{\gamma_{\omega}}, \mathbf{A}_{\gamma_{\omega}}, \mathbf{B}_{\gamma_{\omega}}\right)$ of dimension $n_{\omega}$, respectively. Since they are observable, $\mathcal{O}_{\gamma}$ has full rank and $\operatorname{rank}\left(\mathcal{O}_{S}\right)=\operatorname{rank}(\Psi)$, implying that observability of the system (10) is independent from the statistical model as long as it is observable. This fundamentally enables us to use generic higher order statistical models for $\mathbf{a}$ and $\boldsymbol{\omega}$ to better characterize the motion, instead of the constant speed model used in [41]-[44]. Furthermore, we have the following Lemma:

Lemma 2. The matrix $\mathcal{O}_{S}$ in (24) has full column rank if and only if the matrix

$$
\left[\begin{array}{ccc:c}
\lfloor\boldsymbol{\beta}\rfloor & \mathbf{0} & \mathbf{0} & \mathbf{0} \\
{ }^{2} \mathcal{G}_{1} \mathcal{G}_{\boldsymbol{\theta}} & { }_{n_{1}} \mathcal{G}_{\mathbf{c}}^{\mathbf{h}_{1}} & { }_{n_{1}} \mathcal{G}_{\mathbf{a}}^{\mathbf{h}_{1}} & { }_{n_{1}} \mathcal{G}_{\boldsymbol{\omega}}^{\mathbf{h}_{1}} \\
\hdashline \mathbf{0} & \mathbf{0} & \mathbf{0} & { }_{n} \mathcal{G}_{\boldsymbol{\omega}}^{\mathbf{h}_{2}}
\end{array}\right]=\left[\begin{array}{c:c}
\mathcal{O}_{S_{1}} & \bullet \\
\hdashline \mathbf{0} & \mathcal{O}_{S_{2}}
\end{array}\right]
$$

has full column rank.

Proof. Simplifying $\Psi$ in (24) by the following 3 steps: 1) Since $\mathbf{h}_{2}=\mathbf{R}^{T} \mathbf{e}_{1}=\boldsymbol{\beta}$, it can be shown that $\nabla_{\boldsymbol{\theta}} \mathcal{L}_{\mathbf{f}_{0} \cdots \mathbf{f}_{0}}^{k} \mathbf{h}_{2}$ is in the form of $\mathbf{K}_{k}\lfloor\boldsymbol{\beta}\rfloor$, hence the sub-matrix ${ }_{n}^{1} \mathcal{G}_{\boldsymbol{\theta}} \mathbf{h}_{2}$ in $\boldsymbol{\Psi}$ can be completely eliminated by the preceding row $\lfloor\boldsymbol{\beta}\rfloor ; 2$ ) Eliminate all $\bullet$ elements and the two $\mathbf{I}_{3}$ at $(6,7)$ and $(8,9)$ using the identity matrix in the preceding row or column; 3) Exchange

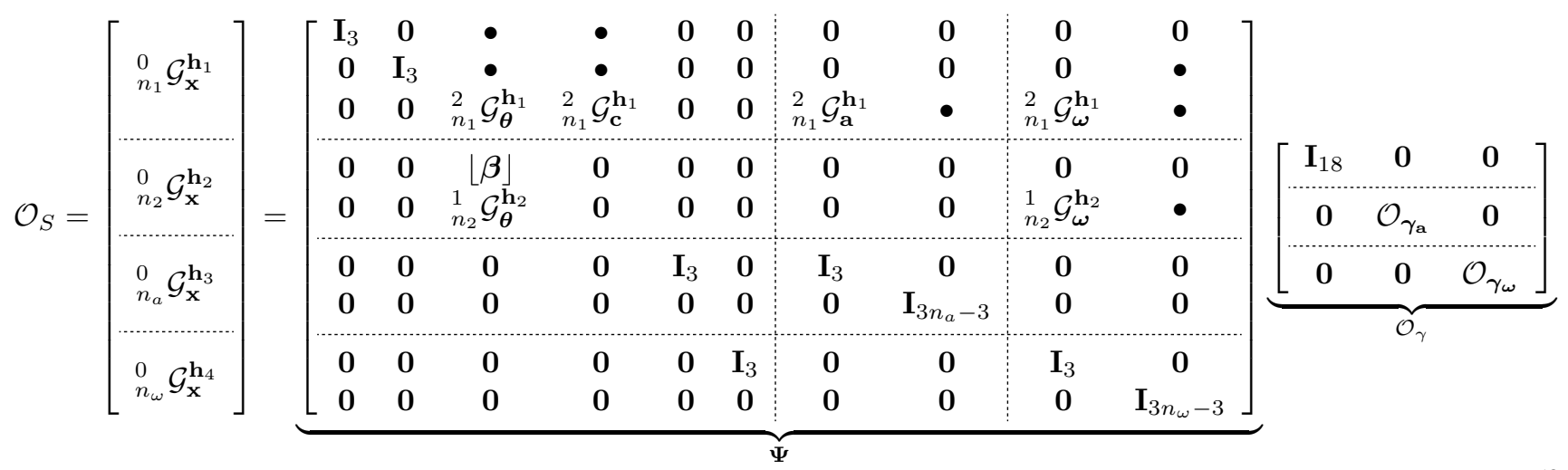


row 3 and 4. Since matrix (block) row transformations do not change its column rank, the lemma statement holds.

Lemma 2 implies that observability of system (10) generally depends on the initial attitude $\mathbf{R}$, parameter $\mathbf{c}$, acceleration $\mathbf{a}$ and its higher derivatives $\dot{\mathbf{a}}, \ddot{\mathbf{a}}, \cdots$, and angular velocity $\boldsymbol{\omega}$ and its higher derivatives $\dot{\boldsymbol{\omega}}, \ddot{\boldsymbol{\omega}}, \cdots$. While this looks more conservative than the input formulation where the observability matrix $\mathcal{O}_{I}$ has full column rank for all initial states $\mathbf{x}$, we should notice that $\mathcal{O}_{I}$ is obtained by assuming the input $\mathbf{a}_{m}$ and $\boldsymbol{\omega}_{m}$ can take arbitrary values in its domain [46]. This corresponds to choosing a value for each of $\mathbf{a}, \boldsymbol{\omega}$ and their higher order derivatives in the state formulation such that the first two block columns of (26) have full column rank, which is true with trivial derivations. This implies that the kinematics states in the state formulation could be observed by properly driving the system with $\mathbf{a}, \boldsymbol{\omega}$ and their higher order derivatives. Furthermore, we could prove that the extended states $\gamma_{\mathbf{a}}$ and $\gamma_{\omega}$ are also observable by showing the full state formulation (10) is locally weakly observable (i.e., $\mathcal{O}_{S}$ in (24) has full column rank) if the system has sufficient excitation. More precisely, we introduce the following definition to characterize the set where the system has insufficient excitation.

Definition 1. A subset $U \subset \mathbb{R}^{n}$ is said to be thin if it contains no open subset $V \subset \mathbb{R}^{n}$.

An equivalent statement for Definition 1 is that for any point $\mathbf{x} \in U$, any open neighborhood $V \subset \mathbb{R}^{n}$ of $\mathbf{x}$ contains points not in $U$. Intuitively, a thin set does not span in every direction of $\mathbb{R}^{n}$. Fig. 7 shows a few examples.

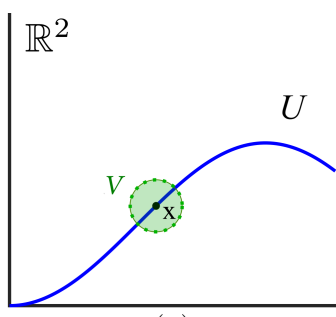

(a)

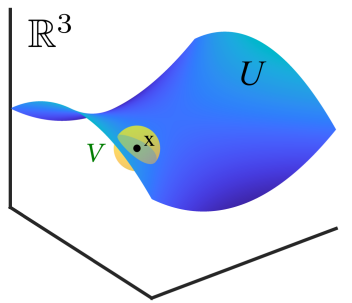

(c)

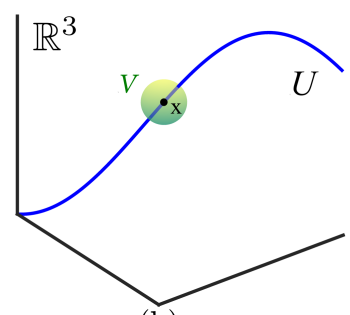

(b)

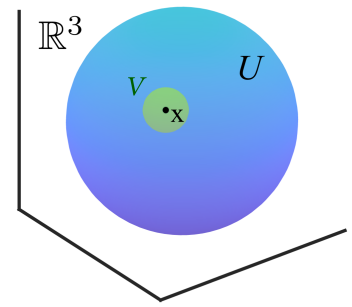

(d)
Fig. 7. Examples for thin subsets: A curve in $\mathbb{R}^{2}$ (a), a curve in $\mathbb{R}^{3}$ (b), and a surface in $\mathbb{R}^{3}$ (c) are thin since they cannot contain any open subset $V$ in the respective $\mathbb{R}^{n}$; (d) A ball in $\mathbb{R}^{3}$ is not thin since it contains an open subset (e.g., a smaller ball) $V \subset \mathbb{R}^{3}$.

Proposition 1. If matrix $\mathcal{O}_{S_{1}}$ in (26) is rank-deficient, the initial states $\gamma_{\mathbf{a}}$ and $\gamma_{\boldsymbol{\omega}}$ (or equivalently $\boldsymbol{\omega}^{(k)}, \mathbf{a}^{(k)}$ ) must lie on a thin subset of $\mathbb{R}^{n_{a}+n_{\omega}}$.

See Appendix. A for proof.
Proposition 2. If matrix $\mathcal{O}_{S_{2}}$ in (26) is rank-deficient, then $\boldsymbol{\omega}^{(k)}$ is parallel to $\boldsymbol{\beta}$, i.e., $\boldsymbol{\omega}^{(k)} \| \boldsymbol{\beta}$, for all $k \geq 0$.

See Appendix. B for proof.

From (26), it is seen that if $\mathcal{O}_{S_{1}}$ and $\mathcal{O}_{S_{1}}$ both has full column rank, $\mathcal{O}_{S}$ has full column rank. Then, the above two propositions imply that the system observability depends on $\boldsymbol{\omega}^{(k)}$ and $\mathbf{a}^{(k)}$ (or equivalently the extended state $\gamma_{\mathbf{a}}$ and $\gamma_{\boldsymbol{\omega}}$ ) only. Moreover, since the subset on which $\mathcal{O}_{S_{1}}$ and $\mathcal{O}_{S_{1}}$ hence $\mathcal{O}_{S}$ are rank-deficient is thin, the probability of the system initial states lying exactly on it is very low (e.g., due to the existence of noises). For the rest majority of $\mathbb{R}^{n_{a}+n_{\omega}}$, i.e., $\boldsymbol{\omega}^{(k)}$ and $\mathbf{a}^{(k)}$ have sufficient excitation, the system is locally weakly observable. This is exactly the same case for the input formulation where sufficient excitation in $\boldsymbol{\omega}$ and $\mathbf{a}$ is required. The only difference is that the $\boldsymbol{\omega}$ and a appear as input instead of state.

\section{Simulation Results}

In order to validate the proposed framework, we simulate a trajectory for the UAV in Fig. 5. In the simulation, the UAV takes off at $2 s$ and stays at $5 \mathrm{~m}$ height, then lands slowly after $12 \mathrm{~s}$. The UAV is driven by sinusoidal acceleration and angular acceleration of multiple frequencies, which give sufficient excitation to the system. The IMU is installed at $\mathbf{c}=\left[\begin{array}{lll}0.5 \mathrm{~m} & 0.5 \mathrm{~m} & 0.5 \mathrm{~m}\end{array}\right]^{T}$ offset to the position sensor. The UAV position, acceleration, and angular velocity are added with white Gaussian noises to produce the simulated measurements. Fig. 8 shows the position and attitude of the IMU in the simulation where Euler angles (Yaw, Pitch and Roll) following the extrinsic Z-Y-X order are used to represent the attitude.

We compare the state estimation results of two error-state EKFs, one based on the state formulation (10) and the other based on the commonly used input formulation (9) (i.e., referred to as "Normal EKF"). For the state formulation, we use a 4-th order integrator as in (2) to model the acceleration and angular velocity independently. Fig. 8 and Fig. 9 show the comparison results, where the kinematics states (i.e, position

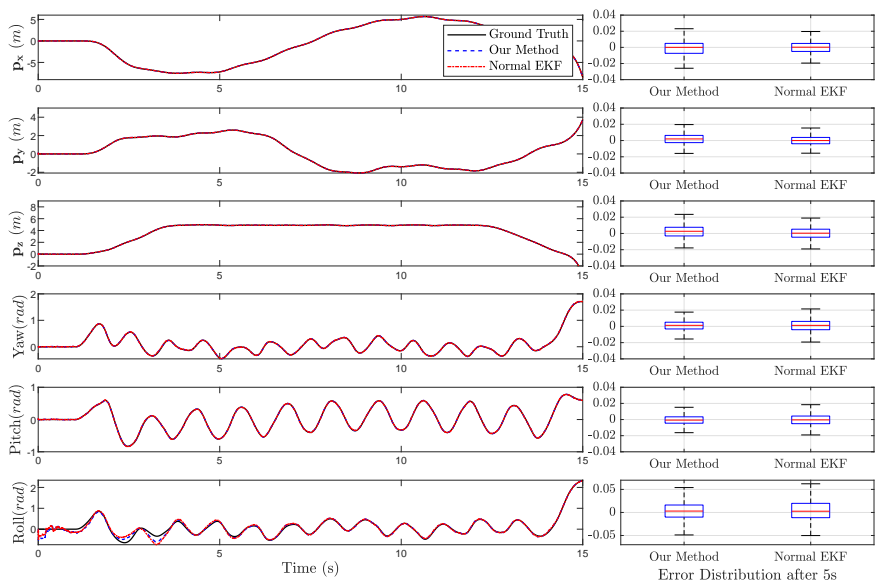

Fig. 8. The estimation results and error distribution of position and attitude in the simulation. 


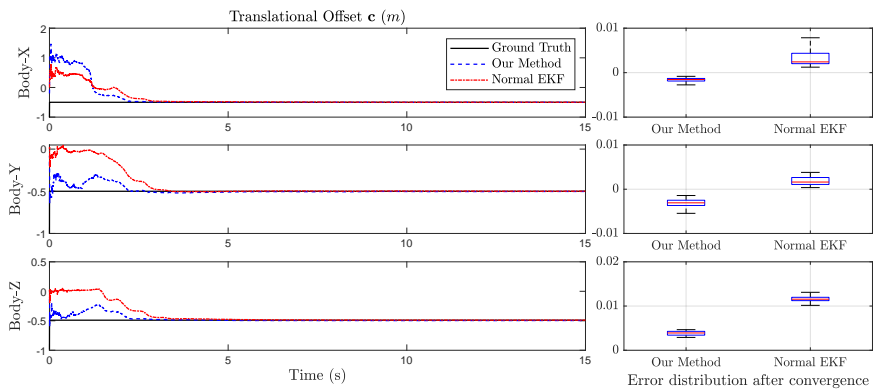

Fig. 9. The estimation results of the translational offset $\mathbf{c}$ in simulation.
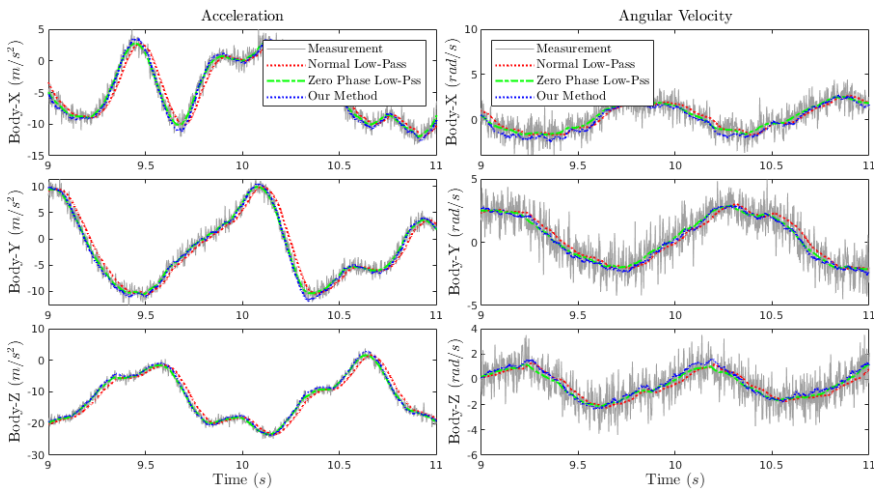

Fig. 10. Comparison between our method, normal low-pass filter and zerophase low-pass filter in the simulation.

$\mathbf{p}$, velocity $\mathbf{v}$, attitude $\mathbf{R}$, and biases $\mathbf{b}_{\mathbf{a}}, \mathbf{b}_{\boldsymbol{\omega}}$ ) and extrinsic parameter $\mathbf{c}$ are shown because the input formulation can only estimate these states. From Fig. 8 and Fig. 9, we can see that both EKFs converge to the ground truth values, and the estimation errors after convergence have comparable variances. This verifies that our method can achieve similar estimation performance on kinematics state as that of the input formulation.

A major advantage of the proposed state formulation is its ability to estimate the acceleration and angular velocity. To evaluate the estimation performance, we compare its estimation results with a first-order Butterworth low-pass filter (referred to as "Normal Low-Pass") and a non-causal zerophase low-pass filter (referred to as "Zero Phase Low-Pass") [68] directly applied on the accelerometer and gyro measurements removing biases. The Butterworth low-pass filter is designed following section III-C such that it attains a noise attenuation level similar to EKF, and the zero-phase filter runs the Butterworth filter in its forward direction [68]. All the results are shown in Fig. 10, where we can see that all the three filters achieve similar noise attenuation w.r.t. the raw measurements. Moreover, it is apparent that the normal lowpass filter has a considerable delay $(40 \mathrm{~ms}$ ) while the filtered results of the zero-phase filter and our EKF are very close to the ground truth values. It should be noted that our EKF is causal, being able to run in an online fashion, while the zerophase filter is not, and that the 4-th order integrator model is not an exact model of the ground true sinusoidal acceleration and angular velocities. In conclusion, a statistical model is robust to capture the UAV dynamic motion and an EKF based on it can estimate the dynamics state with a delay as low as a non-causal zero-phase filter.

\section{EXPERIMENTAL VALIDATION}

This section supplies experimental results to verify the proposed approach. Two applications are demonstrated: the first one is the same to the simulation in Section VI: estimating both the kinematics state and dynamics states (i.e., acceleration and angular velocity) while simultaneously calibrating the offset between the the position reference point and IMU (see Fig. 5). The experiment of the first application is conducted on an UAV, an UGV and a handheld platforms (see Fig. 1) to show the versatility of the proposed statistical model. The second application is to calibrate the extrinsic parameters (both rotation and translation) between two IMUs. Finally, running time of the proposed method is evaluated.

\section{A. Simultaneous States Estimation and Extrinsic Calibration of POS/IMU Sensors}

As shown in Fig. 1, the system is configured with tracker balls of a motion capture system to provide the position and orientation measurement, and an onboard IMU sensor to provide the acceleration and angular velocity measurements. The two sensors (i.e., motion capture system and IMU) are pre-calibrated to align their reference frames. This system is identical to Fig. 5 and hence the proposed approach in section IV and its implementation in section VI can be directly applied here. The three systems are moved randomly in the 3D space (for UAV and handheld platform) or on the floor (for UGV) to obtain sufficient excitation, their trajectories are shown in Fig. 11. In each scenario, we compare the estimated kinematics state with that of the normal EKF based on the input formulation and the estimated acceleration and angular velocity with that of zero-phase low-pass filters.

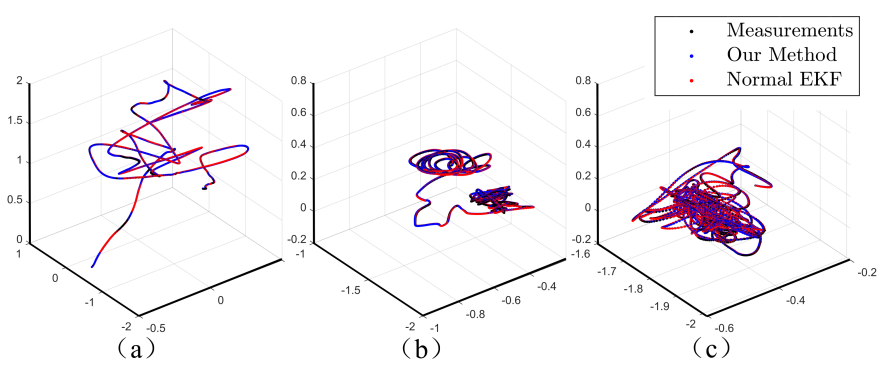

Fig. 11. The trajectories in three validation experiments of the first application: (a) the UAV flight experiment; (b) the UGV experiment; (c) the handheld experiment.

The estimation performance of the kinematics states and extrinsic parameters are shown in Fig. 12, where the first three figures compare the measured position $\mathbf{p}_{m}$ to the position prediction $(\widehat{\mathbf{p}}+\mathbf{R} \widehat{\mathbf{c}})$ from the two EKFs. It is seen that in all these three platforms, both EKFs have comparable means and variances in position prediction errors. With the pre-calibration between the motion capture system and the onboard IMU, we 

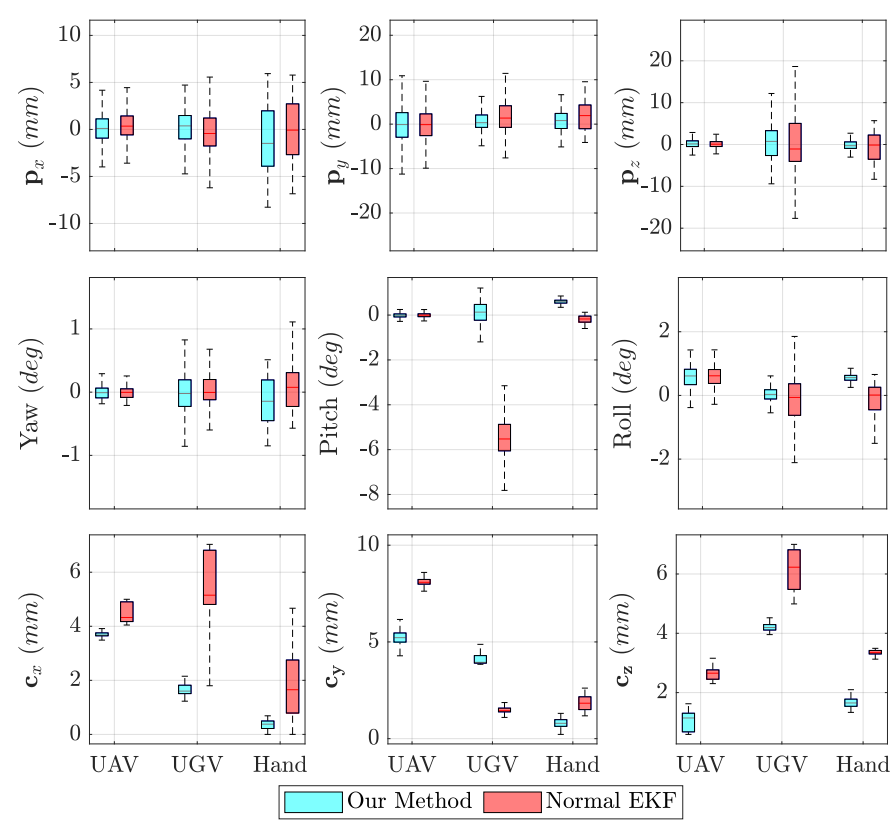

Fig. 12. The error distribution of position $\left(\mathbf{p}_{x} \mathbf{p}_{y} \mathbf{p}_{z}\right)$, attitude (Yaw Pitch Roll) and translational offset $\left(\mathbf{c}_{x} \mathbf{c}_{y} \mathbf{c}_{z}\right)$ after convergence in UGV, UAV and handheld experiments.
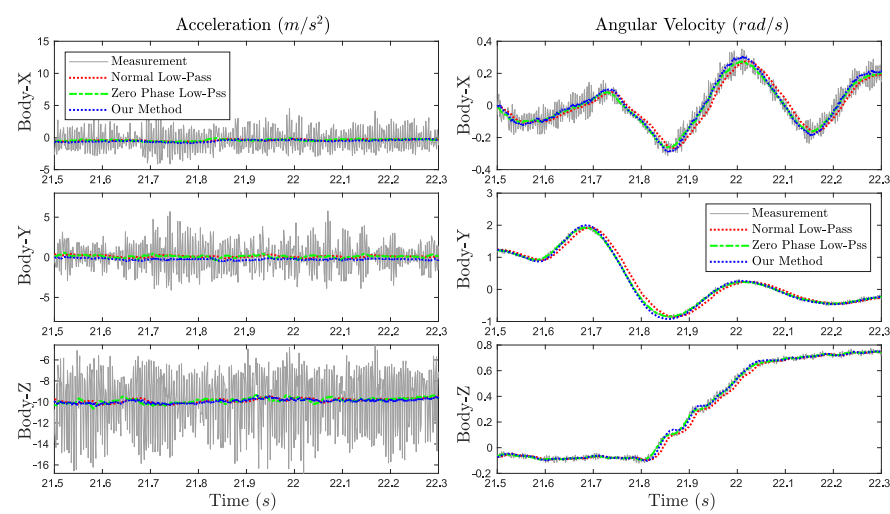

Fig. 13. The comparison of acceleration and angular velocity estimations with a normal low-pass filter outputs and a non-causal zero-phase low-pass filter outputs, for the UAV flight experiment.

also extract the full attitude measurement from the motion capture system and use it as the ground truth to evaluate attitude estimation performance. Shown in the middle three subplots of Fig. 12, overall, the two EKFs have comparable mean and variance. For the UGV case, the pitch error of our method is significantly better than that of the normal EKF. It is also observed some residual errors in attitude estimation, which might be due to the calibration error between mocap and onboard IMU. Fig. 12 further shows the calibration error of the translational offset $\mathbf{c}$ by comparing the extrinsic estimation from the two EKFs to hand measured values. It can be seen that our method achieves smaller errors overall, the difference between the two EKFs are very small (within $5 \mathrm{~mm}$ ), which is below the precision of hand measurements. These results show that the EKF based on the proposed statistical model achieves an estimation performance comparable to (and occasionally better than) conventional input formulation. Besides
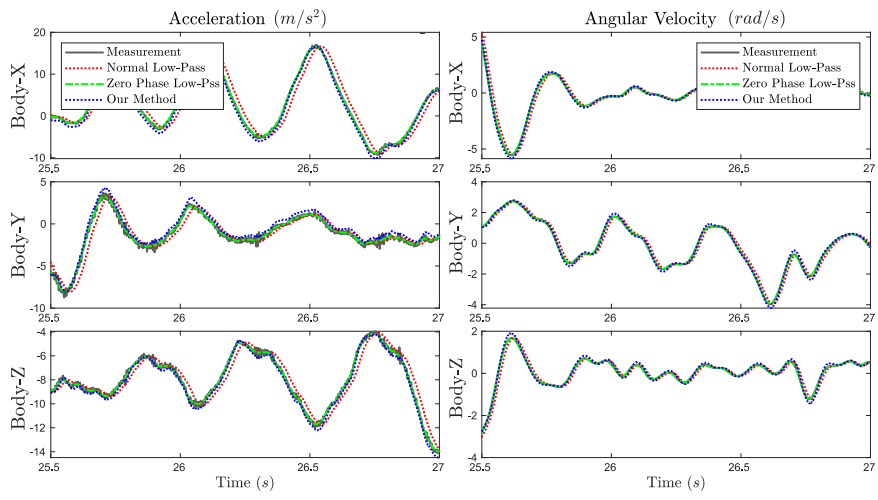

Fig. 14. The comparison of acceleration and angular velocity estimations with a normal low-pass filter outputs and a zero-phase low-pass filter outputs, for the UGV experiment.
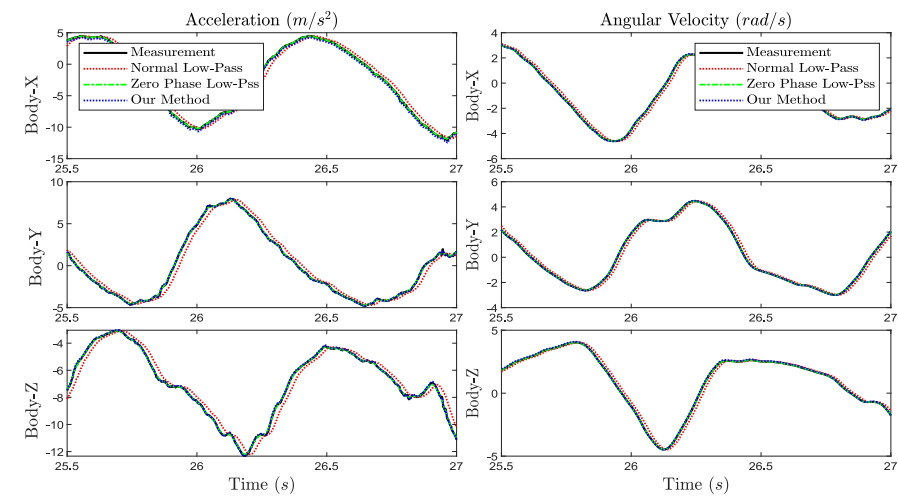

Fig. 15. The comparison of acceleration and angular velocity estimations with a normal low-pass filter outputs and a zero-phase low-pass filter outputs, for the handheld experiment.

the estimation of kinematics state, Fig. 13, Fig. 14 and 15 show the filter results of acceleration and angular velocity on the three platforms. It can be seen that our method has much lower filtering delay (e.g., $21 \mathrm{~ms}$ less for the angular velocity of UAV) in all cases than the normal low-pass filter while they both achieve similar level of noise attenuation. The filtering performance is comparable to a non-causal zero-phase filter. For the acceleration of the UAV case, the noise suppression is easily seen but not the filtering delay due to the small and relative steady body accelerations. This is because the UAV undergoes low speed motions in a confined indoor area (see Fig. 11 (a)), in this case, the rotor drag is very small $[55,69]$ and the specific force acting on the UAV is only the rotor thrust which is always along the body $Z$ direction. We could further exploit this property to calibrate the offset between the IMU position and the center of gravity: if such offset is present and the UAV undergoes rotation w.r.t. its center of gravity, accelerations along body $X$ and $Y$ will show up. This is however beyond the scope of this paper. For the UGV and handheld platforms, the reduced delay in acceleration estimation of our method relative to the low-pass filter is obvious due to the drastic acceleration change. These three experiments draw consistent conclusions: 1) The two EKFs have comparable performance in estimating kinematics state and extrinsic parameters; 2) Our proposed method can 


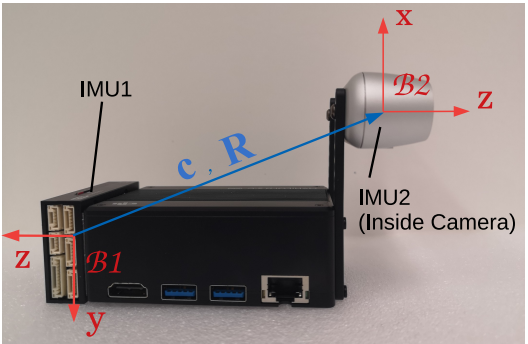

Fig. 16. System configuration of inter IMU calibration.

additionally filter the dynamics states such as acceleration and its filtering performance is comparable to a non-causal zerophase filter.

\section{B. Inter IMU calibration}

The multi-IMU case exists in many robotic applications, for example many UAV platform has back-up IMUs to obtain redundancies. Fig. 16 shows one such system with two IMUs. Extrinsic parameters (both translation and rotation) of the two IMUs must be calibrated to register their data to the same coordinate frame. This is possible with only onboard IMU measurements: since the two IMUs measure the same acceleration vector and angular velocity vector under their respective body frame, the relative rotation could be calibrated, and the relative translation could be calibrated since it relates the linear acceleration of one IMU to the angular acceleration of the other. It can be seen that to calibrate the relative rotation, either angular velocity or linear acceleration of the two IMUs are required, and to calibrate the relative translation, the angular acceleration (hence angular velocity) of one IMU and the linear acceleration of the other are required. This gives us a variety of possible combinations, in this case study, we make use of the angular velocity and the linear acceleration of IMU1 and the linear acceleration of IMU2.

To demonstrate how the proposed statistical model can be used to estimate the linear and angular acceleration, thus enabling online calibration of the two IMUs' extrinsic parameters, we first build a state space model. Referring to Fig. 16, we denote $\mathcal{B} 1$ and $\mathcal{B} 2$ as the body frame of the two IMUs, respectively. The $\mathbf{c}$ and $\mathbf{R}$ in Fig.16 denotes the relative translation and rotation between IMU1 and IMU2, respectively. Choose $\mathcal{B} 1$ as the reference frame and model its linear acceleration $\mathbf{a}_{1}$ and angular acceleration $\tau_{1}$ as two independent statistical model (1) with state vector $\gamma_{\mathbf{a}_{1}}$ and $\gamma_{\tau_{1}}$, respectively. Then, the state equation is:

$$
\begin{aligned}
& \dot{\gamma}_{\mathbf{a}_{1}}=\mathbf{A}_{\gamma_{\mathrm{a}_{1}}} \gamma_{\mathbf{a}_{1}}+\mathbf{B}_{\gamma_{\mathbf{a}_{1}}} \mathbf{w}_{\gamma_{\mathbf{a}_{1}}}, \quad \mathbf{a}_{1}=\mathbf{C}_{\gamma_{\mathbf{a}_{1}}} \gamma_{\mathbf{a}_{1}} \\
& \dot{\gamma}_{\tau_{1}}=\mathbf{A}_{\gamma_{\tau_{1}}} \gamma_{\tau_{1}}+\mathbf{B}_{\gamma_{\tau_{1}}} \mathbf{w}_{\gamma_{\tau_{1}}}, \quad \boldsymbol{\tau}_{1}=\mathbf{C}_{\gamma_{\tau_{1}}} \gamma_{\tau_{1}} \\
& \dot{\boldsymbol{\omega}}_{1}=\tau_{1}, \quad \dot{\mathrm{b}}_{\boldsymbol{\omega}_{1}}=\mathbf{w}_{\mathbf{b}_{\boldsymbol{\omega}_{1}}} \text {, } \\
& \dot{\mathbf{b}}_{\mathbf{a}_{1}}=\mathbf{w}_{\mathbf{b}_{\mathbf{a}_{1}}}, \quad \dot{\mathbf{b}}_{\mathbf{a}_{2}}=\mathbf{w}_{\mathbf{b}_{\mathbf{a}_{2}}} \\
& \dot{\mathbf{c}}=\mathbf{0}, \quad \dot{\mathbf{R}}=\mathbf{0}
\end{aligned}
$$

where $\mathbf{b}_{\mathbf{a}_{i}}$ and $\mathbf{b}_{\boldsymbol{\omega}_{i}}$ are the biases of the $i$-th IMU. It should be noticed that in the above model, we modeled the angular acceleration (instead of angular velocity as in Section VII. A) as a statistical model because it needs to be estimated. The angular acceleration model is then augmented with one additional deterministic integrator to obtain the angular velocity.

The measurement vector is $\mathbf{y}^{T}=\left[\begin{array}{lll}\boldsymbol{\omega}_{m_{1}}^{T} & \mathbf{a}_{m_{1}}^{T} & \mathbf{a}_{m_{2}}^{T}\end{array}\right]$ with components defined below.

$$
\begin{aligned}
\boldsymbol{\omega}_{m_{1}} & =\boldsymbol{\omega}_{1}+\mathbf{b}_{\boldsymbol{\omega}_{1}}+\mathbf{n}_{\boldsymbol{\omega}_{1}} \\
\mathbf{a}_{m_{1}} & =\mathbf{a}_{1}+\mathbf{b}_{\mathbf{a}_{1}}+\mathbf{n}_{\mathbf{a}_{1}} \\
\mathbf{a}_{m_{2}} & =\mathbf{R}\left(\mathbf{a}_{1}+\left\lfloor\boldsymbol{\omega}_{1}\right\rfloor^{2} \mathbf{c}+\left\lfloor\boldsymbol{\tau}_{1}\right\rfloor \mathbf{c}\right)+\mathbf{b}_{\mathbf{a}_{2}}+\mathbf{n}_{\mathbf{a}_{2}}
\end{aligned}
$$

Rewriting the state space model with state equation (27) and output equation (28) into a more compact form as below

$$
\begin{aligned}
\dot{\mathbf{x}} & =\mathbf{f}(\mathbf{x}, \mathbf{w}) \\
\mathbf{y} & =\mathbf{h}(\mathbf{x})
\end{aligned}
$$

where the state vector is

$$
\mathbf{x}^{T}=\left[\boldsymbol{\gamma}_{\mathbf{a}_{1}}^{T}, \boldsymbol{\gamma}_{\boldsymbol{\tau}_{1}}^{T}, \boldsymbol{\omega}_{1}^{T}, \mathbf{b}_{\boldsymbol{\omega}_{1}}^{T}, \mathbf{b}_{\mathbf{a}_{1}}^{T}, \mathbf{b}_{\mathbf{a}_{2}}^{T}, \mathbf{c}^{T}, \mathbf{R}^{T}\right]
$$

If define a constant vector $\overline{\mathrm{x}}$ such that

$$
\begin{aligned}
& \overline{\mathbf{x}}^{T}=\left[\overline{\boldsymbol{\gamma}}_{\mathbf{a}_{1}}^{T}, \mathbf{0}^{T}, \mathbf{0}^{T}, \mathbf{0}^{T}, \overline{\mathbf{b}}_{\mathbf{a}_{1}}^{T}, \overline{\mathbf{b}}_{\mathbf{a}_{2}}^{T}, \mathbf{0}^{T}, \mathbf{0}^{T}\right] \\
& \text { s.t. } \quad \mathbf{A}_{\gamma_{\mathbf{a}_{1}}} \bar{\gamma}_{\mathbf{a}_{1}}=\mathbf{0}, \quad \mathbf{C}_{{\mathbf{a}_{1}}_{\mathbf{a}_{1}}} \bar{\gamma}_{\mathbf{a}_{1}}+\overline{\mathbf{b}}_{\mathbf{a}_{1}}=\mathbf{0}
\end{aligned}
$$

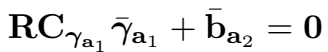

then it is easy to show that the system (29) is invariant to the initial state $\overline{\mathbf{x}}$. i.e., $\check{\mathbf{x}}=\mathbf{x}+\overline{\mathbf{x}}$ satisfy

$$
\begin{aligned}
& \dot{\mathbf{x}}=\mathbf{f}(\check{\mathbf{x}}, \mathbf{w}) \\
& \mathbf{y}=\mathbf{h}(\check{\mathbf{x}})
\end{aligned}
$$

which implies that $\check{\mathbf{x}}$ would produce the same output trajectory (ignoring noises) as that of $\mathbf{x}$ and hence these two states cannot be distinguished. This means that the state space model with state equation (27) and output equation (28) is not a minimal realization.

Further investigating the structure of (30), we find that $\overline{\mathbf{x}}=$ $\mathbf{0}$ if and only if the subsystem

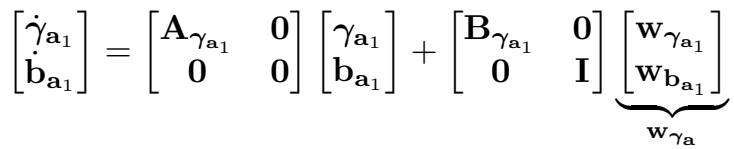

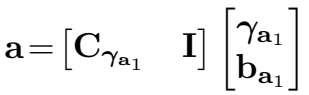

is observable. One sufficient condition for (31) to be observable is choosing $\mathbf{A}_{\boldsymbol{\gamma}_{\mathrm{a}_{1}}}$ to be non-singular. However, this is too conservative (i.e., it limits the representation capability of the statistical model) and unnecessary: when (31) is unobservable, it implies that the statistical model used to capture $\mathbf{a}$, the biased acceleration, has redundant states that should be eliminated when designing the statistical model in the first place. Removing the redundant states can be achieved by transforming (31) into the Kalman canonical form as below [56].

$$
\begin{aligned}
{\left[\begin{array}{c}
\dot{\gamma}_{\mathrm{a}} \\
\bullet
\end{array}\right] } & =\left[\begin{array}{cc}
\mathbf{A}_{\gamma_{\mathrm{a}}} & 0 \\
\bullet & \bullet
\end{array}\right]\left[\begin{array}{c}
\gamma_{\mathrm{a}} \\
\bullet
\end{array}\right]+\left[\begin{array}{c}
\mathbf{B}_{\gamma_{\mathrm{a}}} \\
\bullet
\end{array}\right] \mathbf{w}_{\gamma_{\mathrm{a}}} \\
\mathbf{a} & =\left[\begin{array}{ll}
\mathbf{C}_{\gamma_{\mathrm{a}}} & \mathbf{0}
\end{array}\right]\left[\begin{array}{c}
\gamma_{\mathbf{a}} \\
\bullet
\end{array}\right]
\end{aligned}
$$

where $\left(\mathbf{C}_{\gamma_{\mathrm{a}}}, \mathbf{A}_{\gamma_{\mathrm{a}}}\right)$ is observable subsystem and $\bullet$ 's are terms belong to the unobservable subspace. Then it is sufficient to keep only the observable state component $\gamma_{\mathbf{a}}$ in (32) as it fully 
represents the biased acceleration a hence the measurement $\mathbf{a}_{m_{1}}$. Furthermore, from the output equation of (31), we have $\mathbf{a}_{1}=\mathbf{C}_{\gamma_{\mathbf{a}_{1}}} \boldsymbol{\gamma}_{\mathbf{a}_{1}}=\mathbf{a}-\mathbf{b}_{\mathbf{a}_{1}}$. Hence, the measurement $\mathbf{a}_{m_{2}}$ can be fully represented by the biased acceleration $\mathbf{a}$ and the relative bias $\mathbf{b}_{\mathbf{a}}=\mathbf{b}_{\mathbf{a}_{2}}-\mathbf{R} \mathbf{b}_{\mathbf{a}_{1}}$. Finally dropping the subscript 1,2 for the sake of notation simplicity, the state equation (27) can be reduced to the following minimal realization.

$$
\begin{gathered}
\dot{\mathbf{b}}_{\mathbf{a}}=\mathbf{w}_{\mathbf{b}_{\mathbf{a}}}, \dot{\mathbf{b}}_{\boldsymbol{\omega}}=\mathbf{w}_{\mathbf{b}_{\boldsymbol{\omega}}}, \dot{\boldsymbol{\omega}}=\boldsymbol{\tau}, \dot{\mathbf{c}}=\mathbf{0}, \dot{\mathbf{R}}=\mathbf{0} \\
\dot{\gamma}_{\mathbf{a}}=\mathbf{A}_{\gamma_{\mathbf{a}}} \gamma_{\mathbf{a}}+\mathbf{B}_{\gamma_{\mathbf{a}}} \mathbf{w}_{\gamma_{\mathbf{a}}}, \dot{\gamma}_{\boldsymbol{\tau}}=\mathbf{A}_{\gamma_{\tau}} \gamma_{\boldsymbol{\tau}}+\mathbf{B}_{\gamma_{\tau}} \mathbf{w}_{\gamma_{\tau}}
\end{gathered}
$$

where $\mathbf{w}_{\mathbf{b}_{\mathbf{a}}}=\mathbf{w}_{\mathbf{b}_{\mathbf{a}_{2}}}-\mathbf{R} \mathbf{w}_{\mathbf{b}_{\mathbf{a}_{1}}}$ is a relative noise seen in $\mathcal{B} 2$ and the output equation is

$$
\begin{aligned}
& \mathbf{a}=\mathbf{C}_{\boldsymbol{\gamma}_{\mathbf{a}}} \boldsymbol{\gamma}_{\mathbf{a}}, \quad \boldsymbol{\tau}=\mathbf{C}_{\boldsymbol{\tau}_{\boldsymbol{\tau}}} \gamma_{\boldsymbol{\tau}} \\
& \boldsymbol{\omega}_{m_{1}}=\boldsymbol{\omega}+\mathbf{b}_{\boldsymbol{\omega}}+\mathbf{n}_{\boldsymbol{\omega}}, \mathbf{a}_{m_{1}}=\mathbf{a}+\mathbf{n}_{\mathbf{a}}, \\
& \mathbf{a}_{m_{2}}=\mathbf{R}\left(\mathbf{a}+\lfloor\boldsymbol{\omega}\rfloor^{2} \mathbf{c}+\lfloor\boldsymbol{\tau}\rfloor \mathbf{c}\right)+\mathbf{b}_{\mathbf{a}}+\mathbf{n}_{\mathbf{a}}
\end{aligned}
$$

Lemma 3. The nonlinear system with state equation

$$
\begin{gathered}
\dot{\mathrm{b}}_{\mathbf{a}}=\mathbf{0}, \dot{\mathrm{b}}_{\boldsymbol{\omega}}=\mathbf{0}, \dot{\mathbf{c}}=\mathbf{0}, \dot{\mathbf{R}}=\mathbf{0} \\
\dot{\boldsymbol{\omega}}=\boldsymbol{\tau}, \dot{\gamma}_{\boldsymbol{\tau}}=\mathbf{A}_{\gamma_{\tau}} \gamma_{\boldsymbol{\tau}}, \dot{\gamma}_{\mathbf{a}}=\mathbf{A}_{\gamma_{\mathbf{a}}} \gamma_{\mathbf{a}}
\end{gathered}
$$

where $\left(\mathbf{C}_{\boldsymbol{\gamma}_{\mathbf{a}}}, \mathbf{A}_{\boldsymbol{\gamma}_{\mathbf{a}}}\right)$ and $\left(\mathbf{C}_{\boldsymbol{\gamma}_{\tau}}, \mathbf{A}_{\boldsymbol{\gamma}_{\tau}}\right)$ are both observable and output equation

$$
\begin{aligned}
& \mathbf{h}_{1}=\mathbf{R}\left(\mathbf{a}+\lfloor\boldsymbol{\omega}\rfloor^{2} \mathbf{c}+\lfloor\boldsymbol{\tau}\rfloor \mathbf{c}\right)+\mathbf{b}_{\mathbf{a}} \\
& \mathbf{h}_{2}=\boldsymbol{\omega}+\mathbf{b}_{\boldsymbol{\omega}}, \\
& \mathbf{h}_{3}=\mathbf{a}
\end{aligned}
$$

is locally weakly observable if $\mathbf{c} \neq \mathbf{0}$ and the system initial states $\boldsymbol{\omega}^{(k)}$ and $\mathbf{a}^{(k)}, k \geq 0$ have sufficient excitation.

Proof. See Appendix. C.

Lemma 3 implies that the overall system observability is independent from the exact form of statistical models used for $\boldsymbol{\tau}$ and $\mathbf{a}$ as long as they are observable. This allows us to choose generic higher order statistical models that better characterize the motion without affecting the observability. Furthermore, Lemma 3 enables us to design an error state EKF similar to Section IV-D to estimate the extrinsic parameters $\mathbf{c}, \mathbf{R}$ online.

With the mathematical formulation and theoretical proof on its observability, we now proceed to the experiment verification. The sensor suite containing two IMUs is shaken in all 6 degree of freedom (DOF) to give the system sufficient excitation. To evaluate the effectiveness of the proposed statistical models, the output of the two statistical models $\left(\mathbf{C}_{\gamma_{\mathbf{a}}}, \mathbf{A}_{\gamma_{\mathbf{a}}}\right)$ and $\left(\mathbf{C}_{\gamma_{\tau}}, \mathbf{A}_{\gamma_{\tau}}\right)$, which are a and $\boldsymbol{\tau}$, respectively, are compared to the "ground truth". Since $\mathbf{a}$ is the acceleration with bias of IMU1, we compare it to the ground truth obtained by filtering the accelerometer measurements of IMU1 via a non-causal low-pass filter aimed to attenuate the measurement noise. Similar, $\tau$ means the angular acceleration of IMU1, we compare it to the ground truth obtained by numerically differentiating the gyro measurements of IMU1 and then filtering it via a non-causal low-pass filter. Fig 17 shows the comparison results. It is seen that our estimation closely track the results obtained by non-causal filter without a noticeable delay. There is a slight overshoot when the acceleration suddenly changes
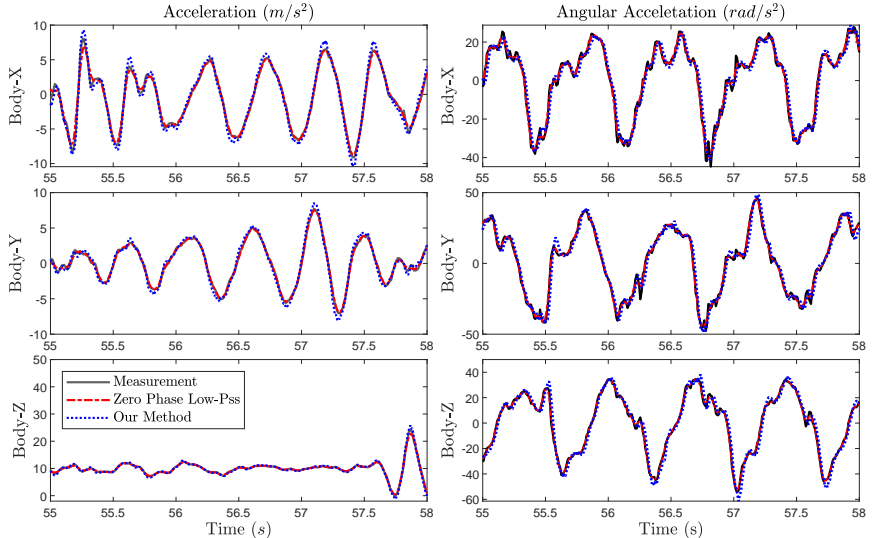

Fig. 17. The estimated acceleration with bias and the estimated angular acceleration of IMU1.
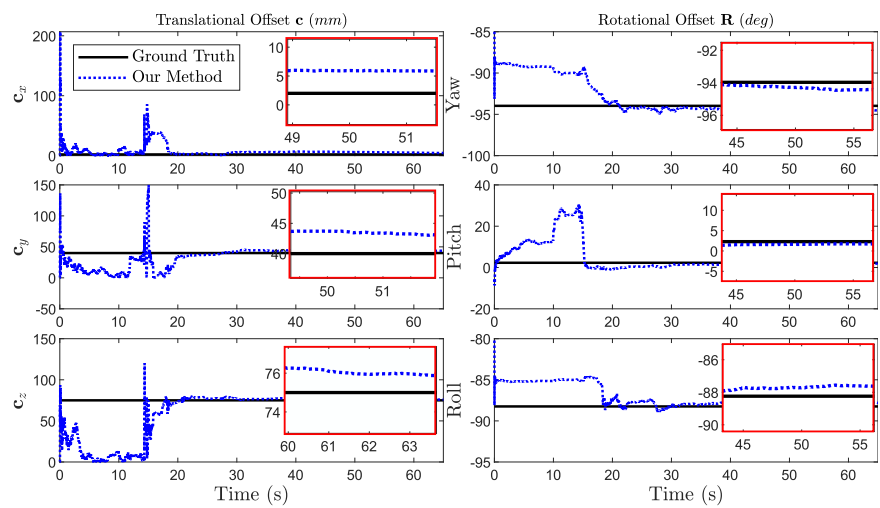

Fig. 18. The estimation of translational offset and rotational offset.

its direction. This is due to the non-exact model (i.e., 4-th order integrator) being used for the handheld motion. This slight overshoot is also in agreement with the frequency domain analysis in Section. III-C. Fig. 18 shows the relative translation c and rotation $\mathbf{R}$ between the two IMUs. The ground truth is obtained by hand measurements for translation and CAD design for rotation. It can be seen that the estimated offset (both translation and rotation) quickly converges. The final converged error is $5 \mathrm{~mm}$ in translation and 1 degree in rotation, which are below the precision of hand measurements.

\section{Running Time}

An apparent drawback of the statistical model-based state estimation approach is that the system order is increased by the dimension of the statistical model. This will increase computation time of the EKF. Taking the application in Section VII-A as an example, if a 4-th order integrator (2) is used for each channel of acceleration and angular velocity, the system dimension will increase by 24 orders. To verify the real-time running performance of the proposed approach, we implement the error-state $\mathrm{EKF}$ in $\mathrm{C}++$. As a comparison, the running time of the normal EKF based on input formulation is also tested. The experiments run on a personal computer with processor of Intel Core i7-8700. As shown in Table. I, the state formulation EKF takes more than 3 times computation time than the input formulation, which is expected because of the 
increased system dimension. Nevertheless, the total running time is below $0.04 \mathrm{~ms}$, which is only a fraction of other modules (e.g., planning) and is sufficient for real-time running for most robotic applications. This can be further reduced by using the statistical model only when necessary. For example, when linear acceleration is required to be estimated while the angular velocity is not, then a hybrid input and state formulation could be used where the gyro measurements are formulated as input and the accelerometer measurements are formulated as outputs with an augmented statistical model.

TABLE I

COMPARISON OF THE AVERAge RUn TIME

\begin{tabular}{cccc}
\hline Methods & Predict & Update & Total \\
\hline State formulation & $0.0174 \mathrm{~ms}$ & $0.0200 \mathrm{~ms}$ & $0.0374 \mathrm{~ms}$ \\
\hline Input formulation & $0.0050 \mathrm{~ms}$ & $0.0056 \mathrm{~ms}$ & $0.0106 \mathrm{~ms}$ \\
\hline
\end{tabular}

\section{CONCLUSION}

This paper proposed to modeling a robot's dynamics by a statistical model. Such a statistical model is robust and versatile, being applicable to different robotic systems without accurately modeling the robot's specific first principle dynamics. By augmenting the statistical model to the robot's kinematic equation, it enables to simultaneously estimate the dynamics state with low latency in addition to the kinematics states and extrinsic parameters. Extensive simulations and experiments on different robots platforms for different applications were conducted and the results supports the proposed methods. The augmented system requires more computations due to the increased state dimension, but the overall computation time is still sufficiently small for real-time implementations. The proposed method is suitable for applications where dynamic states (e.g., translational or angular accelerations) are needed in real-time.

Besides the practical contribution, this paper also proposed new theoretical tools to ease the observability analysis of robotic systems operating on manifolds and in high dimension. A new paradigm which calculates Lie derivatives and partial differentiation directly on the respective manifolds is developed. This new paradigm is simpler, more natural and scalable to systems of high dimensions. Furthermore, a novel thin set concept was introduced to characterize the unobservable subspace of the system states. These lay theoretical foundations for observability analysis for robotic systems of high dimension.

\section{APPENDIX}

Theorem 4. Let $p(\mathbf{x})$ be a multivariate polynomial of $\mathbf{x}=$ $\left[x_{1}, \cdots, x_{n}\right]^{T} \in \mathbb{R}^{n}$. If $p(\mathbf{x})$ is not constant zero, then

$$
U=\left\{\mathbf{x} \in \mathbb{R}^{n} \mid p(\mathbf{x})=0\right\}
$$

is a thin set in $\mathbb{R}^{n}$ by Definition 1 .

Proof. Assume $U$ is not thin, then there must exist an open subset $V \subset \mathbb{R}^{n}$ contained in $U$ such that

$$
p(\mathbf{x})=0, \forall \mathbf{x} \in V .
$$

Since this equation holds on the entire open subset $V$ on which $p(\mathbf{x})$ is infinitely differentiable, we have

$$
\frac{\partial^{m} p(\mathbf{x})}{\partial t_{m} \cdots \partial t_{1}}=0 ; m \geq 0, t_{i} \in\left\{x_{1}, \cdots, x_{n}\right\}, \forall \mathbf{x} \in V .
$$

Since $p(\mathbf{x})$ is a multivariate polynomial, it can be expressed as

$$
p(\mathbf{x})=\sum_{i=1}^{m} a_{i}\left(\prod_{j=1}^{n} x_{j}^{k_{i j}}\right) ; k_{i j} \in\{0,1, \cdots\}
$$

Substituting $p(\mathbf{x})$ into the above partial differentiation and letting $m_{i}=\sum_{j=1}^{n} k_{i j}$ leads to

$$
\frac{\partial^{m_{i}} p(\mathbf{x})}{\partial x_{n}^{k_{i n}} \cdots \partial x_{1}^{k_{i 1}}}=a_{i}=0 ; i=1, \cdots, m
$$

and $p(\mathbf{x}) \equiv 0$, which contradicts with $p(\mathbf{x})$ not being constant zero.

Lemma 5. Let $\boldsymbol{\Omega}=\lfloor\boldsymbol{\omega}\rfloor^{2}+\left\lfloor\boldsymbol{\omega}^{(1)}\right\rfloor$. If $i \geq 2$ is the first order of derivative such that $\boldsymbol{\omega}^{(i)} \neq \mathbf{0}$ and $j>i$ is the first order of derivative such that $\boldsymbol{\omega}^{(j)} \nVdash \boldsymbol{\omega}^{(i)}$, then the matrix

$$
\boldsymbol{\Theta}=\left[\begin{array}{l}
\boldsymbol{\Omega}^{(i-1)} \\
\boldsymbol{\Omega}^{(j-1)}
\end{array}\right]
$$

has full column rank.

Proof. It is shown that $\boldsymbol{\Omega}^{(m)}=\sum_{k=0}^{m} \mathrm{C}_{k}^{m}\left\lfloor\boldsymbol{\omega}^{(k)}\right\rfloor\left\lfloor\boldsymbol{\omega}^{(m-k)}\right\rfloor+$ $\left\lfloor\boldsymbol{\omega}^{(m+1)}\right\rfloor$. And as indicated in the description of this Lemma,

$$
\begin{cases}\boldsymbol{\omega}^{(k)}=\mathbf{0} & k \in\{0,1, \cdots, i-1\} \\ \boldsymbol{\omega}^{(i)} \neq \mathbf{0} & \\ \boldsymbol{\omega}^{(k)}=\mu_{k} \boldsymbol{\omega}^{(i)} & k \in\{i+1, \cdots, j-1\} \\ \boldsymbol{\omega}^{(j)} \nVdash \boldsymbol{\omega}^{(i)} & \end{cases}
$$

Then

$$
\begin{aligned}
& \boldsymbol{\Omega}^{(i-1)}=\left\lfloor\boldsymbol{\omega}^{(i)}\right\rfloor \\
& \boldsymbol{\Omega}^{(j-1)}= \begin{cases}\Lambda_{j-1}^{i}\left\lfloor\boldsymbol{\omega}^{(i)}\right\rfloor^{2}+\left\lfloor\boldsymbol{\omega}^{(j)}\right\rfloor & 2 i \leq j-1 \\
\left\lfloor\boldsymbol{\omega}^{(j)}\right\rfloor & \text { otherwise }\end{cases}
\end{aligned}
$$

where $\Lambda_{j-1}^{i}=\sum_{k=i}^{j-1-i} C_{k}^{j-1} \mu_{k} \mu_{j-1-k}$.

Assuming $\boldsymbol{\Theta}$ does not have full column rank, then $\exists \mathbf{v} \neq \mathbf{0}$, s.t. $\boldsymbol{\Theta} \cdot \mathbf{v}=\mathbf{0}$. Then we have $\mathbf{v} \| \boldsymbol{\omega}^{(i)}$ from $\boldsymbol{\Omega}^{(i-1)} \mathbf{v}=\mathbf{0}$ and $\mathbf{v} \| \boldsymbol{\omega}^{(j)}$ from $\boldsymbol{\Omega}^{(j-1)} \mathbf{v}=\mathbf{0}$. This is, however, contradictory to the condition that $\boldsymbol{\omega}^{(j)} \nVdash \boldsymbol{\omega}^{(i)}$. Thus $\boldsymbol{\Theta}$ has full column rank.

\section{A. Proof of Proposition 1}

Proof. Let $\mathbf{b}=\mathbf{a}+\mathbf{\Omega} \mathbf{c}$, the acceleration at the position reference point and seen in the body frame, and $\mathbf{g}_{m}=\mathbf{R}^{T} \mathbf{h}_{1}^{(m+2)}$, the $(m+2)$-th time derivative of the position reference point and seen in the body frame, we can derive, by induction, that

$$
\begin{aligned}
\mathbf{g}_{0} & =\mathbf{b} \\
\mathbf{g}_{m} & =\mathbf{b}^{(m)}+\sum_{k=0}^{m-1} \boldsymbol{\alpha}_{k}^{m} \mathbf{g}_{k} ; m \geq 1
\end{aligned}
$$


where $\boldsymbol{\alpha}_{k}^{m}$ is defined recursively as below:

$$
\begin{aligned}
\boldsymbol{\alpha}_{0}^{1} & =\lfloor\boldsymbol{\omega}\rfloor \\
\boldsymbol{\alpha}_{k}^{m+1} & = \begin{cases}\dot{\boldsymbol{\alpha}}_{k}^{m}-\boldsymbol{\alpha}_{k}^{m}\lfloor\boldsymbol{\omega}\rfloor & k=0 \\
\dot{\boldsymbol{\alpha}}_{k}^{m}-\boldsymbol{\alpha}_{k}^{m}\lfloor\boldsymbol{\omega}\rfloor+\boldsymbol{\alpha}_{k-1}^{m} & 1 \leq k \leq m-1 \\
\lfloor\boldsymbol{\omega}\rfloor+\boldsymbol{\alpha}_{k-1}^{m} & k=m\end{cases}
\end{aligned}
$$

It is seen that $\boldsymbol{\alpha}_{k}^{m}$ are polynomials of $\boldsymbol{\omega}^{(k)}$ and $\mathbf{g}_{m}$ are polynomials of $\boldsymbol{\omega}^{(k)}, \mathbf{a}^{(k)}$, and c. Hence,

$$
\begin{array}{r}
\nabla_{\boldsymbol{\theta}} \mathbf{g}_{m}=\mathbf{0} ; \nabla_{\mathbf{c}} \mathbf{g}_{m}=\mathbf{\Omega}^{(m)}+\sum_{k=0}^{m-1} \boldsymbol{\alpha}_{k}^{m}\left(\nabla_{\mathbf{c}} \mathbf{g}_{k}\right) \\
\nabla_{\mathbf{a}} \mathbf{g}_{m}= \begin{cases}\mathbf{I}_{3} & m=0 \\
\sum_{k=0}^{m-1} \boldsymbol{\alpha}_{k}^{m}\left(\nabla_{\mathbf{a}} \mathbf{g}_{k}\right) & m \geq 1\end{cases}
\end{array}
$$

By definition,

$$
\left(\mathcal{L}_{\mathbf{f}_{0}}^{1} \mathbf{h}_{1}\right)(\mathbf{x})=\dot{\mathbf{h}}_{1}(\mathbf{x}), \text { subject to } \dot{\mathbf{x}}=\mathbf{f}_{0}(\mathbf{x})
$$

where $\mathbf{f}_{0}(\mathbf{x})$ abbreviates $(25)$, we have

$$
\begin{aligned}
\left(\mathcal{L}_{\mathbf{f}_{0} \cdots \mathbf{f}_{0}}^{m+2} \mathbf{h}_{1}\right)(\mathbf{x}) & =\mathbf{h}_{1}^{(m+2)}(\mathbf{x}), \text { subject to } \dot{\mathbf{x}}=\mathbf{f}_{0}(\mathbf{x}) \\
& =\mathbf{R g}_{m}
\end{aligned}
$$

Then, $\mathcal{O}_{S_{1}}$ in $(26)$ is detailed as:

$$
\begin{aligned}
\mathcal{O}_{S_{1}}= & {\left[\begin{array}{ccc}
\lfloor\boldsymbol{\beta}\rfloor & \mathbf{0} & \multicolumn{1}{c}{\mathbf{0}} \\
2{ }_{n_{1}} \mathcal{G}_{\boldsymbol{\theta}}^{\mathbf{h}_{1}} & { }_{n_{1}}^{2} \mathcal{G}_{\mathbf{c}}^{\mathbf{h}_{1}} & { }_{n_{1}}^{2} \mathcal{G}_{\mathbf{a}}^{\mathbf{h}_{1}}
\end{array}\right] } \\
{ }_{n_{1}} \mathcal{G}_{\boldsymbol{\theta}}^{\mathbf{h}_{1}}= & {\left[\begin{array}{c}
\vdots \\
\nabla_{\boldsymbol{\theta}}\left(\mathcal{L}_{\mathbf{f}_{0} \cdots \mathbf{f}_{0}}^{m+2} \mathbf{h}_{1}\right) \\
\vdots
\end{array}\right]=\left[\begin{array}{c}
\vdots \\
-\mathbf{R}\left\lfloor\mathbf{g}_{m}\right\rfloor \\
\vdots
\end{array}\right] } \\
{ }_{n_{1}} \mathcal{G}_{\mathbf{c}} \mathbf{h}_{1}= & {\left[\begin{array}{c}
\nabla_{\mathbf{c}}\left(\mathcal{L}_{\mathbf{f}_{0} \cdots \mathbf{f}_{0}}^{m+2} \mathbf{h}_{1}\right) \\
\vdots \\
\vdots \\
\vdots \\
n_{1} \mathcal{G}_{\mathbf{a}}^{\mathbf{h}_{1}}=
\end{array}\right]=\left[\begin{array}{c}
\vdots \\
\mathbf{R} \nabla_{\mathbf{c}} \mathbf{g}_{m} \\
\vdots \\
\nabla_{\mathbf{a}}\left(\mathcal{L}_{\mathbf{f}_{0} \cdots \mathbf{f}_{0}}^{m+2} \mathbf{h}_{1}\right) \\
\vdots
\end{array}\right]=\left[\begin{array}{c}
\vdots \\
\mathbf{R} \nabla_{\mathbf{a}} \mathbf{g}_{m} \\
\vdots
\end{array}\right] }
\end{aligned}
$$

where $m=0,1, \cdots, n_{1}-2$. Since the rotation matrix $\mathbf{R}$ does not affect the rank, we have

$$
\mathcal{O}_{S_{1}} \sim\left[\begin{array}{ccc}
\lfloor\boldsymbol{\beta}\rfloor & \mathbf{0} & \mathbf{0} \\
-\left\lfloor\mathbf{g}_{0}\right\rfloor & \boldsymbol{\Omega}^{(0)} & \mathbf{I}_{3} \\
\vdots & \vdots & \vdots \\
-\left\lfloor\mathbf{g}_{m}\right\rfloor & \mathbf{\Omega}^{(m)}+\mathbf{\Pi}_{\mathbf{c}}^{m} & \boldsymbol{\Pi}_{\mathbf{a}}^{m} \\
\vdots & \vdots & \vdots
\end{array}\right]
$$

where $m=1,2, \cdots, n_{1}-2, \boldsymbol{\Pi}_{\mathbf{c}}^{m}=\sum_{k=0}^{m-1} \boldsymbol{\alpha}_{k}^{m}\left(\nabla_{\mathbf{c}} \mathbf{g}_{k}\right)$ and $\boldsymbol{\Pi}_{\mathbf{a}}^{m}=\sum_{k=0}^{m-1} \boldsymbol{\alpha}_{k}^{m}\left(\nabla_{\mathbf{a}} \mathbf{g}_{k}\right)$ are linearly dependent on preceding rows and can therefore be eliminated by Gaussian elimination. Hence,

$$
\mathcal{O}_{S_{1}} \sim\left[\begin{array}{ccc}
\lfloor\boldsymbol{\beta}\rfloor & \mathbf{0} & \mathbf{0} \\
-\left\lfloor\mathbf{g}_{0}\right\rfloor & \boldsymbol{\Omega}^{(0)} & \mathbf{I}_{3} \\
\vdots & \vdots & \vdots \\
-\left\lfloor\mathbf{g}_{m}\right\rfloor+\sum_{k=0}^{m-1} \boldsymbol{\alpha}_{k}^{m}\left\lfloor\mathbf{g}_{k}\right\rfloor & \boldsymbol{\Omega}^{(m)} & \mathbf{0} \\
\vdots & \vdots & \vdots
\end{array}\right]
$$

Since column 3 only contains an identity matrix, the other terms in row 2 can be eliminated to zero by column transformation. Therefore, the $\mathcal{O}_{S_{1}}$ has full column rank if and only if the following sub-matrix $\mathcal{O}_{S_{1}}^{E}$ have full column rank.

$$
\mathcal{O}_{S_{1}}^{E}=\left[\begin{array}{ccc}
\lfloor\boldsymbol{\beta}\rfloor & \mathbf{0} & \mathbf{0} \\
\vdots & \vdots & \vdots \\
-\left\lfloor\mathbf{g}_{m}\right\rfloor+\sum_{k=0}^{m-1} \boldsymbol{\alpha}_{k}^{m}\left\lfloor\mathbf{g}_{k}\right\rfloor & \boldsymbol{\Omega}^{(m)} & \mathbf{0} \\
\vdots & \vdots & \vdots
\end{array}\right] ; m \geq 1
$$

Notice that elements of $\mathcal{O}_{S_{1}}^{E}$ are polynomial functions of $\boldsymbol{\omega}^{(k)}, \mathbf{a}^{(k)}$, and $\mathbf{c}$ for a given $\boldsymbol{\beta}=\mathbf{R}^{T} \mathbf{e}_{1}$, and that $\boldsymbol{\omega}^{(k)}$ and $\mathbf{a}^{(k)}$ are further linearly dependent on $\gamma_{\boldsymbol{\omega}}$ and $\gamma_{\mathbf{a}}$, respectively, thus elements of $\mathcal{O}_{S_{1}}^{E}$ are polynomial functions of the following subset of states:

$$
\mathbf{x}_{\text {sub }}=\left[\begin{array}{c}
\gamma_{\boldsymbol{\omega}} \\
\gamma_{\mathbf{a}} \\
\mathbf{c}
\end{array}\right] \in \mathbb{R}^{n_{\omega}+n_{a}+3}
$$

Denote

$$
\operatorname{Sub}\left(\mathcal{O}_{S_{1}}^{E}\right)
$$

the set of all square matrix whose rows are drawn from $\mathcal{O}_{S_{1}}^{E}$. Since elements of $\mathcal{O}_{S_{1}}^{E}$ are polynomials of $\mathbf{x}_{\text {sub }}$, the determinant $\operatorname{det}(\mathbf{S}), \forall \mathbf{S} \in \operatorname{Sub}\left(\mathcal{O}_{S_{1}}^{E}\right)$ is also a polynomial function of $\mathbf{x}_{\text {sub }}$. Then if $\mathcal{O}_{S_{1}}^{E}$ is rank-deficient, there must have

$$
\operatorname{det}(\mathbf{S})\left(\mathbf{x}_{\text {sub }}\right)=0, \forall \mathbf{S} \in \operatorname{Sub}\left(\mathcal{O}_{S_{1}}^{E}\right)
$$

which forms infinite number of equality constraints. For each constraint, $\operatorname{det}(\mathbf{S})\left(\mathbf{x}_{\text {sub }}\right)$ is a polynomial of $\mathbf{x}_{\text {sub }}$ and its roots are either the whole space of $\mathbb{R}^{n_{\omega}+n_{a}+3}$ if $\operatorname{det}(\mathbf{S})\left(\mathbf{x}_{\text {sub }}\right) \equiv 0$ or otherwise lie on a thin subset (see Theorem 4).

Now we show that $\mathcal{O}_{S_{1}}^{E}$ is not constantly rank-deficient by giving a particular sufficient condition as follows.

(i) $i \geq 3$ is the first order of derivative such that $\boldsymbol{\omega}^{(i)} \neq \mathbf{0}$ and $j>i$ is the first order of derivative such that $\boldsymbol{\omega}^{(j)} \nmid \boldsymbol{\omega}^{(i)}$

(ii) $\exists k \in\{1, \cdots, i-2\}$, s.t. $\mathbf{a}^{(k)} \nVdash \boldsymbol{\beta}$

From Lemma 5, condition (i) ensures the matrix

$$
\boldsymbol{\Theta}=\left[\begin{array}{l}
\boldsymbol{\Omega}^{(i-1)} \\
\boldsymbol{\Omega}^{(j-1)}
\end{array}\right]
$$


has full column rank and that $\boldsymbol{\Omega}^{(k)}=\mathbf{0}$. Then the matrix $\mathcal{O}_{S_{1}}^{E}$ can be reduced to

$$
\mathcal{O}_{S_{1}}^{E} \sim\left[\begin{array}{c:c}
\Psi & 0 \\
\vdots & \vdots \\
\bullet & \Theta \\
\vdots & \vdots
\end{array}\right]
$$

where $\boldsymbol{\Psi}=\left[\begin{array}{c}\lfloor\boldsymbol{\beta}\rfloor \\ -\left\lfloor\mathbf{a}^{(k)}\right\rfloor\end{array}\right]$.

Then, condition (ii) ensures $\boldsymbol{\Psi}$ has full column rank and hence the matrix $\mathcal{O}_{S_{1}}^{E}$ has full column rank. This means $\exists \mathbf{S} \in$ $\operatorname{Sub}\left(\mathcal{O}_{S_{1}}^{E}\right)$ and $\overline{\mathbf{x}}_{\text {sub }} \in \mathbb{R}^{n_{\omega}+n_{a}+3}$ such that $\operatorname{det}(\mathbf{S})\left(\overline{\mathbf{x}}_{\text {sub }}\right) \neq$ 0 . Moreover, since $\operatorname{det}(\mathbf{S})\left(\mathbf{x}_{\text {sub }}\right)$ is a continuous function of $\mathbf{x}_{\text {sub }}$, so if $\operatorname{det}(\mathbf{S})\left(\mathbf{x}_{\text {sub }}\right) \neq 0$ at point $\overline{\mathbf{x}}_{\text {sub }}$, it must be so in a neighbour of that point, meaning that there are infinite number of submatrix $\mathbf{S} \in \mathcal{O}_{\text {sub }}$ whose determinant is not constant zero and hence the solution of each $\operatorname{det}(\mathbf{S})=0$ lies on a thin subset of $\mathbb{R}^{n_{\omega}+n_{a}+3}$. As a result, the states $\mathbf{x}_{\text {sub }}$ satisfying $\mathcal{O}_{\text {sub }}$ being rank-deficient is the joint of the infinite number of thin subset and the resultant solution space is even thinner, i.e., a thin subset in $\mathbb{R}^{n_{\omega}+n_{a}+3}$.

\section{B. Proof of Proposition 2}

Proof. Recall that $\mathbf{h}_{2}=\mathbf{R}^{T} \mathbf{e}_{1} \triangleq \boldsymbol{\beta}$, the matrix $\mathcal{O}_{S_{2}}=$ ${ }_{n_{2}} \mathcal{G}_{\boldsymbol{\omega}} \mathbf{h}_{2}=\left[\begin{array}{lll}\nabla_{\boldsymbol{\omega}} \boldsymbol{\beta}^{(1)} & \ldots & \nabla_{\boldsymbol{\omega}} \boldsymbol{\beta}^{\left(n_{2}\right)}\end{array}\right]^{T}$. Since $\dot{\boldsymbol{\beta}}=\lfloor\boldsymbol{\omega}\rfloor^{T} \boldsymbol{\beta}=$ $\lfloor\boldsymbol{\beta}\rfloor \boldsymbol{\omega}$, each term $\nabla_{\boldsymbol{\omega}} \boldsymbol{\beta}^{(m)}, m \in\left\{1, \cdots, n_{2}\right\}$ can be computed as:

$$
\begin{aligned}
\nabla_{\boldsymbol{\omega}} \boldsymbol{\beta}^{(m)} & =\frac{\partial}{\partial \boldsymbol{\omega}} \frac{d^{m} \boldsymbol{\beta}}{d t^{m}}=\frac{\partial}{\partial \boldsymbol{\omega}} \frac{d^{m-1}(\dot{\boldsymbol{\beta}})}{d t^{m-1}}=\frac{\partial}{\partial \boldsymbol{\omega}} \frac{d^{m-1}\left(\lfloor\boldsymbol{\omega}\rfloor^{T} \boldsymbol{\beta}\right)}{d t^{m-1}} \\
= & \frac{\partial}{\partial \boldsymbol{\omega}} \sum_{i=0}^{m-1} \mathrm{C}_{i}^{m-1}\left\lfloor\boldsymbol{\omega}^{(i)}\right\rfloor^{T} \boldsymbol{\beta}^{(m-1-i)} \\
= & \sum_{i=1}^{m} \mathrm{C}_{i-1}^{m-1}\left\lfloor\boldsymbol{\beta}^{(m-i)}\right\rfloor \frac{\partial}{\partial \boldsymbol{\omega}^{(i-1)}} \\
& \quad+\sum_{i=1}^{m} \mathrm{C}_{i-1}^{m-1}\left\lfloor\boldsymbol{\omega}^{(i-1)}\right\rfloor^{T} \frac{\partial}{\partial \boldsymbol{\omega}} \boldsymbol{\beta}^{(m-i)} \\
= & \left\lfloor\boldsymbol{\beta}^{(m-1)}\right\rfloor+\sum_{i=1}^{m} \underbrace{\mathrm{C}_{i-1}^{m-1}\left\lfloor\boldsymbol{\omega}^{(i-1)}\right\rfloor^{T} \nabla_{\boldsymbol{\omega}} \boldsymbol{\beta}^{(m-i)}}_{\mathbf{K}_{i}}
\end{aligned}
$$

Notice that terms $\mathbf{K}_{i}, 0 \leq i \leq m-1$ can be eliminated by preceding rows using the Gaussian elimination and $\mathbf{K}_{m}=\mathbf{0}$, the only left term is $\left\lfloor\boldsymbol{\beta}^{(m-1)}\right\rfloor$. Therefore

$$
\mathcal{O}_{S_{2}} \sim\left[\begin{array}{c}
\lfloor\boldsymbol{\beta}\rfloor \\
\cdots \\
\left\lfloor\boldsymbol{\beta}^{\left(n_{2}-1\right)}\right\rfloor
\end{array}\right]
$$

Since $\mathcal{O}_{S_{2}}$ is rank-deficient, then $\exists \mathbf{v} \neq \mathbf{0}$ such that $\mathcal{O}_{S_{2}} \mathbf{v}=$ $\mathbf{0}$, which implies $\left\lfloor\boldsymbol{\beta}^{(m)}\right\rfloor \mathbf{v}=\mathbf{0}, \forall m \geq 0$. In particular, let $m=$ 0 , then $\boldsymbol{\beta} \| \mathbf{v}$; let $m=1,\left\lfloor\boldsymbol{\beta}^{(1)}\right\rfloor \mathbf{v}=\mathbf{0} \Longrightarrow\left\lfloor\lfloor\boldsymbol{\omega}\rfloor^{T} \boldsymbol{\beta}\right\rfloor \boldsymbol{\beta}=$ $\mathbf{0} \Longrightarrow \boldsymbol{\omega} \| \boldsymbol{\beta}$. Now assume that $\boldsymbol{\omega}^{(m)} \| \boldsymbol{\beta}$ is true for $0 \leq m \leq k-1$, then

$$
\begin{aligned}
& \left\lfloor\boldsymbol{\beta}^{(k+1)}\right\rfloor \boldsymbol{\beta}=\mathbf{0} \Rightarrow\left\lfloor\frac{d^{k} \dot{\boldsymbol{\beta}}}{d t^{k}}\right\rfloor \boldsymbol{\beta}=\mathbf{0} \\
& \Rightarrow \sum_{i=0}^{k}\left\lfloor\left\lfloor\boldsymbol{\omega}^{(i)}\right\rfloor^{T} \boldsymbol{\beta}^{(k-i)}\right\rfloor \boldsymbol{\beta}=\mathbf{0} \\
& \Rightarrow \sum_{i=0}^{k-1}\left(\left\lfloor\left\lfloor\boldsymbol{\omega}^{(i)}\right\rfloor^{T} \boldsymbol{\beta}^{(k-i)}\right\rfloor+\left\lfloor\left\lfloor\boldsymbol{\omega}^{(k)}\right\rfloor^{T} \boldsymbol{\beta}\right\rfloor\right) \boldsymbol{\beta}=\mathbf{0} \\
& \Rightarrow-\sum_{i=0}^{k-1} \underbrace{\left.\left.\left(\left\lfloor\boldsymbol{\omega}^{(i)}\right\rfloor \boldsymbol{\beta}^{(k-i)}\right\rfloor-\left\lfloor\boldsymbol{\beta}^{(k-i)}\right\rfloor \boldsymbol{\omega}^{(i)}\right\rfloor\right)}_{=\mathbf{0}} \boldsymbol{\beta}+\left\lfloor\boldsymbol{\omega}^{(k)}\right\rfloor^{T} \boldsymbol{\beta}\rfloor \boldsymbol{\beta}=\mathbf{0} \\
& \Rightarrow\left\lfloor\left\lfloor\boldsymbol{\omega}^{(k)}\right\rfloor^{T} \boldsymbol{\beta}\right\rfloor \boldsymbol{\beta}=\mathbf{0} \\
& \Rightarrow \boldsymbol{\omega}^{(k)} \| \boldsymbol{\beta}
\end{aligned}
$$

Therefore, if $\mathcal{O}_{S_{2}}$ is rank-deficient, then $\boldsymbol{\omega}^{(k)} \| \boldsymbol{\beta}, \forall k \geq$ 0 .

\section{Proof of Lemma 3}

Proof. Define the state vector $\mathbf{x}$

$$
\mathbf{x}^{T}=\left[\mathbf{b}_{\mathbf{a}}^{T}, \mathbf{b}_{\boldsymbol{\omega}}^{T}, \mathbf{c}^{T}, \mathbf{R}^{T}, \boldsymbol{\omega}^{T}, \boldsymbol{\gamma}_{\boldsymbol{\tau}}^{T}, \boldsymbol{\gamma}_{\mathbf{a}}^{T}\right]
$$

and rewrite the state equation (33) as $\dot{\mathbf{x}}=\mathbf{f}_{0}(\mathbf{x})$, then the observability matrix is computed as

$$
\mathcal{O}=\left[\begin{array}{c}
{ }_{n}^{0} \mathcal{G}_{\mathbf{x}}^{\mathbf{h}_{1}} \\
\hdashline{ }_{n}^{0} \mathcal{G}_{\mathbf{x}}^{\mathbf{h}_{2}} \\
\hdashline{ }_{n} \mathcal{G}_{\mathbf{x}}^{\mathbf{h}_{3}}
\end{array}\right]=\left[\begin{array}{ccccccc}
\mathbf{I}_{3} & \mathbf{0} & \bullet & \bullet & \bullet & \bullet & \mathbf{0} \\
\mathbf{0} & \mathbf{0} & { }_{n} \mathcal{G}_{\mathbf{c}}^{\mathbf{h}_{1}} & { }_{n}^{1} \mathcal{G}_{\boldsymbol{\theta}}^{\mathbf{h}_{1}} & { }_{n} \mathcal{G}_{\boldsymbol{\omega}}^{\mathbf{h}_{1}} & \bullet & \bullet \\
\hdashline \mathbf{0} & \mathbf{I}_{3} & \mathbf{0} & \mathbf{0} & \mathbf{I}_{3} & \mathbf{0} & \mathbf{0} \\
\mathbf{0} & \mathbf{0} & \mathbf{0} & \mathbf{0} & \mathbf{0} & \mathcal{O}_{\gamma_{\tau}} & \mathbf{0} \\
\hdashline \mathbf{0} & \mathbf{0} & \mathbf{0} & \mathbf{0} & \mathbf{0} & \mathbf{0} & \mathcal{O}_{\gamma_{\mathbf{a}}}
\end{array}\right]
$$

which has full column rank if and only if the following submatrix

$$
\mathcal{O}_{\text {sub }}=\left[\begin{array}{l:l:l}
{ }_{n}^{1} \mathcal{G}_{\mathbf{c}}^{\mathbf{h}_{1}} & { }_{n}^{1} \mathcal{G}_{\boldsymbol{\theta}}^{\mathbf{h}_{1}} & { }_{n}^{1} \mathcal{G}_{\boldsymbol{\omega}}^{\mathbf{h}_{1}}
\end{array}\right]
$$

has full column rank.

Let $\boldsymbol{\Omega}=\lfloor\boldsymbol{\omega}\rfloor^{2}+\left\lfloor\boldsymbol{\omega}^{(1)}\right\rfloor$, then

$$
\mathcal{O}_{\mathrm{sub}} \sim\left[\begin{array}{c:c:c}
\boldsymbol{\Omega}^{(1)} & -\left\lfloor\mathbf{a}^{(1)}+\boldsymbol{\Omega}^{(1)} \mathbf{c}\right\rfloor & -\left\lfloor\left\lfloor\boldsymbol{\omega}^{(1)}\right\rfloor \mathbf{c}\right\rfloor-\left\lfloor\boldsymbol{\omega}^{(1)}\right\rfloor\lfloor\mathbf{c}\rfloor \\
\vdots & \vdots & \vdots \\
\boldsymbol{\Omega}^{(n)} & -\left\lfloor\mathbf{a}^{(n)}+\boldsymbol{\Omega}^{(n)} \mathbf{c}\right\rfloor & -\left\lfloor\left\lfloor\boldsymbol{\omega}^{(n)}\right\rfloor \mathbf{c}\right\rfloor-\left\lfloor\boldsymbol{\omega}^{(n)}\right\rfloor\lfloor\mathbf{c}\rfloor
\end{array}\right]
$$

where the sign $\sim$ indicates equivalent rank. Notice that 1$)$ the rank of $\mathcal{O}_{\text {sub }}$ depends on $\boldsymbol{\omega}^{(k)}, \mathbf{a}^{(k)}$ (hence the extended states $\gamma_{\boldsymbol{\tau}}, \gamma_{\mathbf{a}}$, and state $\boldsymbol{\omega}$ ), and $\mathbf{c}$ only and is independent from the bias $\mathbf{b}_{\mathbf{a}}, \mathbf{b}_{\boldsymbol{\omega}}$, and relative rotation $\mathbf{R}$; and moreover 2) elements of $\mathcal{O}_{\text {sub }}$ are polynomial functions of $\boldsymbol{\omega}^{(k)}, k \geq 0, \mathbf{a}^{(k)}, k \geq 1$ and c. Since $\boldsymbol{\omega}^{(k)}, k \geq 1$ are further linearly dependent on $\gamma_{\boldsymbol{\tau}}$ and $\mathbf{a}^{(k)}$ are further linearly dependent on $\gamma_{\mathbf{a}}$, elements of $\mathcal{O}_{\text {sub }}$ are polynomial functions of the following subset of states

$$
\mathbf{x}_{\mathrm{sub}}=\left[\begin{array}{c}
\gamma_{\boldsymbol{\tau}} \\
\gamma_{\mathbf{a}} \\
\boldsymbol{\omega} \\
\mathbf{c}
\end{array}\right] \in \mathbb{R}^{n_{\tau}+n_{a}+6}
$$


Denote

$$
\operatorname{Sub}\left(\mathcal{O}_{\text {sub }}\right)
$$

the set of all square matrix whose rows are drawn from $\mathcal{O}_{\text {sub }}$. Since elements of $\mathcal{O}_{\text {sub }}$ are polynomials of $\mathbf{x}_{\text {sub }}$, the determinant $\operatorname{det}(\mathbf{S}), \forall \mathbf{S} \in \operatorname{Sub}\left(\mathcal{O}_{\text {sub }}\right)$ is also a polynomial function of $\mathbf{x}_{\text {sub }}$. Then if $\mathcal{O}_{\text {sub }}$ is rank-deficient, there must have

$$
\operatorname{det}(\mathbf{S})\left(\mathbf{x}_{\text {sub }}\right)=0, \forall \mathbf{S} \in \operatorname{Sub}\left(\mathcal{O}_{\text {sub }}\right) .
$$

which forms infinite number of equality constraints for $\mathbf{x}_{\text {sub }}$. For each constraint, $\operatorname{det}(\mathbf{S})\left(\mathbf{x}_{\text {sub }}\right)$ is a polynomial of $\mathbf{x}_{\text {sub }}$ and its roots are either the whole space of $\mathbb{R}^{n_{\tau}+n_{a}+6}$ if $\operatorname{det}(\mathbf{S})\left(\mathbf{x}_{\text {sub }}\right) \equiv 0$ or otherwise lie on a thin subset (see Theorem 4).

Now we show that $\mathcal{O}_{\text {sub }}$ is not constant rank-deficient if $\mathbf{c} \neq \mathbf{0}$ by giving a particular sufficient condition as below.

(i) $i \geq 2$ is the first order of derivative such that $\boldsymbol{\omega}^{(i)} \neq \mathbf{0}$ and $j>i+1$ is the first order of derivative such that $\boldsymbol{\omega}^{(j)} \nVdash \boldsymbol{\omega}^{(i)}, \boldsymbol{\omega}^{(j-1)}=\mathbf{0}$, and $\mathbf{a}^{(i-1)}+\boldsymbol{\Omega}^{(i-1)} \mathbf{c}=\mathbf{a}^{(j-1)}+$ $\mathbf{\Omega}^{(j-1)} \mathbf{c}=\mathbf{0}$.

(ii) $\exists k, l \geq 1$ s.t., $\boldsymbol{\omega}^{(k)}=\boldsymbol{\omega}^{(l)}=\mathbf{0}, \mathbf{a}^{(k)}+\boldsymbol{\Omega}^{(k)} \mathbf{c}$ and $\mathbf{a}^{(l)}+$ $\boldsymbol{\Omega}^{(l)} \mathbf{c}$ are not collinear, and $i-1, j-1, k, l$ are different from each other.

(iii) $\exists m \geq j$ s.t. $\boldsymbol{\omega}^{(m)} \nmid \mathbf{c}$ and $m$ is different from $k, l, i-1$ and $j-1$.

From Lemma 5 , condition (i) ensures the matrix

$$
\boldsymbol{\Theta}=\left[\begin{array}{l}
\boldsymbol{\Omega}^{(i-1)} \\
\boldsymbol{\Omega}^{(j-1)}
\end{array}\right]
$$

has full column rank. Then, the matrix $\mathcal{O}_{\text {sub }}$ can be reduced to

$$
\mathcal{O}_{\mathrm{sub}} \sim\left[\begin{array}{c:c:c}
\vdots & \vdots & \vdots \\
\boldsymbol{\Theta} & \mathbf{0} & \mathbf{0} \\
\bullet & \boldsymbol{\Phi} & \mathbf{0} \\
\bullet & \bullet & \boldsymbol{\Psi} \\
\vdots & \vdots & \vdots
\end{array}\right]
$$

where

$$
\begin{aligned}
& \boldsymbol{\Phi}=\left[\begin{array}{l}
-\left\lfloor\mathbf{a}^{(k)}+\boldsymbol{\Omega}^{(k)} \mathbf{c}\right\rfloor \\
-\left\lfloor\mathbf{a}^{(l)}+\boldsymbol{\Omega}^{(l)} \mathbf{c}\right\rfloor
\end{array}\right] \\
& \boldsymbol{\Psi}=-\left\lfloor\left\lfloor\boldsymbol{\omega}^{(m)}\right\rfloor \mathbf{c}\right\rfloor-\left\lfloor\boldsymbol{\omega}^{(m)}\right\rfloor\lfloor\mathbf{c}\rfloor
\end{aligned}
$$

Then, condition (ii) ensures $\boldsymbol{\Phi}$ has full column rank and condition (iii) ensures $\Psi$ has full rank and hence the matrix $\mathcal{O}_{\text {sub }}$ has full column rank. This means $\exists \mathbf{S} \in \operatorname{Sub}\left(\mathcal{O}_{\text {sub }}\right)$ and $\overline{\mathbf{x}}_{\text {sub }} \in \mathbb{R}^{n_{\tau}+n_{a}+6}$ such that $\operatorname{det}(\mathbf{S})\left(\overline{\mathbf{x}}_{\text {sub }}\right) \neq 0$. Moreover, since 1) $\operatorname{det}(\mathbf{S})\left(\mathbf{x}_{\text {sub }}\right)$ is a continuous function of $\mathbf{x}_{\text {sub }}$, so if $\operatorname{det}(\mathbf{S})\left(\mathbf{x}_{\text {sub }}\right) \neq 0$ at point $\overline{\mathbf{x}}_{\text {sub }}$, it must be so in a neighbour of that point, and 2) the order of $i, j, k, l$ is arbitrary, so there are infinite number of submatrix $\mathbf{S} \in \mathcal{O}_{\text {sub }}$ whose determinant is not constant zero and for each the solution of $\operatorname{det}(\mathbf{S})=0$ lies on a thin subset of $\mathbb{R}^{n_{\tau}+n_{a}+6}$. As a result, the states $\mathbf{x}_{\text {sub }}$ satisfying $\mathcal{O}_{\text {sub }}$ being rank-deficient is the joint of the infinite number of thin subset and the resultant solution space is even thinner. Such a thin subset is generally not satisfied when the system initial states $\boldsymbol{\omega}^{(k)}$ and $\mathbf{a}^{(k)}$ have sufficient excitation.

\section{REFERENCES}

[1] W. Xu and F. Zhang, "Learning pugachev's cobra maneuver for tailsitter uavs using acceleration model," IEEE Robotics and Automation Letters, vol. 5, no. 2, pp. 3452-3459, 2020.

[2] E. J. Smeur, M. Bronz, and G. C. de Croon, "Incremental control and guidance of hybrid aircraft applied to a tailsitter unmanned air vehicle," Journal of Guidance, Control, and Dynamics, vol. 43, no. 2, pp. 274287, 2020.

[3] E. J. Smeur, G. C. de Croon, and Q. Chu, "Cascaded incremental nonlinear dynamic inversion for mav disturbance rejection," Control Engineering Practice, vol. 73, pp. 79-90, 2018.

[4] S. Sieberling, Q. Chu, and J. Mulder, "Robust flight control using incremental nonlinear dynamic inversion and angular acceleration prediction," Journal of guidance, control, and dynamics, vol. 33, no. 6, pp. 17321742,2010

[5] X. Lyu, J. Zhou, H. Gu, Z. Li, S. Shen, and F. Zhang, "Disturbance observer based hovering control of quadrotor tail-sitter vtol uavs using $h_{\infty}$ synthesis," IEEE Robotics and Automation Letters, vol. 3, no. 4, pp. 2910-2917, 2018.

[6] B. Yüksel, C. Secchi, H. H. Bülthoff, and A. Franchi, "A nonlinear force observer for quadrotors and application to physical interactive tasks," in 2014 IEEE/ASME International Conference on Advanced Intelligent Mechatronics. IEEE, 2014, pp. 433-440.

[7] S. H. Jeong, S. Jung, and M. Tomizuka, "Attitude control of a quad-rotor system using an acceleration-based disturbance observer: An empirical approach," in 2012 IEEE/ASME International Conference on Advanced Intelligent Mechatronics (AIM). IEEE, 2012, pp. 916-921.

[8] H. Wang and M. Chen, "Trajectory tracking control for an indoor quadrotor uav based on the disturbance observer," Transactions of the Institute of Measurement and Control, vol. 38, no. 6, pp. 675-692, 2016.

[9] S. J. Lee, S. Kim, K. H. Johansson, and H. J. Kim, "Robust acceleration control of a hexarotor uav with a disturbance observer," in 2016 IEEE 55th Conference on Decision and Control (CDC). IEEE, 2016, pp. 4166-4171.

[10] J. Lee, "High fidelity modelling for high altitude long endurance solar powered aircraft," Ph.D. dissertation, ETH Zürich, 2017.

[11] R. Borobia, G. Sanchez-Arriaga, A. Serino, and R. Schmehl, "Flightpath reconstruction and flight test of four-line power kites," Journal of Guidance, Control, and Dynamics, vol. 41, no. 12, pp. 2604-2614, 2018.

[12] J. Hoff and M. Cook, "Aircraft parameter identification using an estimation-before-modelling technique," The Aeronautical Journal, vol. 100, no. 997, pp. 259-268, 1996.

[13] M. W. Mueller, M. Hehn, and R. D'Andrea, "A computationally efficient motion primitive for quadrocopter trajectory generation," IEEE Transactions on Robotics, vol. 31, no. 6, pp. 1294-1310, 2015.

[14] D. K. Shaeffer, "Mems inertial sensors: A tutorial overview," IEEE Communications Magazine, vol. 51, no. 4, pp. 100-109, 2013.

[15] R. Mahony, T. Hamel, and J.-M. Pflimlin, "Complementary filter design on the special orthogonal group $S O(3)$," in Proceedings of the 44th IEEE Conference on Decision and Control. IEEE, 2005, pp. 14771484.

[16] H. T. Mahony, Robert and J.-M. Pflimlin, "Nonlinear complementary filters on the special orthogonal group," IEEE Transactions on automatic control, vol. 53, no. 5, pp. 1203-1218, 2008.

[17] G. L. Smith, S. F. Schmidt, and L. A. McGee, Application of statistical filter theory to the optimal estimation of position and velocity on board a circumlunar vehicle. National Aeronautics and Space Administration, 1962.

[18] F. L. Markley, "Attitude error representations for kalman filtering," Journal of guidance, control, and dynamics, vol. 26, no. 2, pp. 311$317,2003$.

[19] N. Trawny and S. I. Roumeliotis, "Indirect kalman filter for 3d attitude estimation," University of Minnesota, Dept. of Comp. Sci. \& Eng., Tech. Rep, vol. 2, p. 2005, 2005.

[20] F. L. Markley and J. L. Crassidis, Fundamentals of spacecraft attitude determination and control. Springer, 2014, vol. 33.

[21] J. Sola, "Quaternion kinematics for the error-state kalman filter," arXiv preprint arXiv:1711.02508, 2017.

[22] G. Lu and F. Zhang, "Imu-based attitude estimation in the presence of narrow-band noise," IEEE/ASME Transactions on Mechatronics, vol. 24, no. 2, pp. 841-852, 2019.

[23] F. Caron, E. Duflos, D. Pomorski, and P. Vanheeghe, "Gps/imu data fusion using multisensor kalman filtering: introduction of contextual aspects," Information fusion, vol. 7, no. 2, pp. 221-230, 2006. 
[24] A. Pfister, A. M. West, S. Bronner, and J. A. Noah, "Comparative abilities of microsoft kinect and vicon $3 \mathrm{~d}$ motion capture for gait analysis," Journal of medical engineering \& technology, vol. 38, no. 5, pp. 274-280, 2014.

[25] T. Qin, P. Li, and S. Shen, "Vins-mono: A robust and versatile monocular visual-inertial state estimator," IEEE Transactions on Robotics, vol. 34, no. 4, pp. 1004-1020, 2018.

[26] C. Forster, L. Carlone, F. Dellaert, and D. Scaramuzza, "On-manifold preintegration for real-time visual-inertial odometry," IEEE Transactions on Robotics, vol. 33, no. 1, pp. 1-21, 2016.

[27] A. I. Mourikis and S. I. Roumeliotis, "A multi-state constraint kalman filter for vision-aided inertial navigation," in Proceedings 2007 IEEE International Conference on Robotics and Automation. IEEE, 2007, pp. 3565-3572.

[28] A. Bry, A. Bachrach, and N. Roy, "State estimation for aggressive flight in gps-denied environments using onboard sensing," in 2012 IEEE International Conference on Robotics and Automation. IEEE, 2012, pp. $1-8$.

[29] J. Lin and F. Zhang, "Loam_livox: A fast, robust, high-precision lidar odometry and mapping package for lidars of small fov," arXiv preprint arXiv:1909.06700, 2019.

[30] B. K. Horn and B. G. Schunck, "Determining optical flow," in Techniques and Applications of Image Understanding, vol. 281. International Society for Optics and Photonics, 1981, pp. 319-331.

[31] A. Soloviev and A. J. Rutkowski, "Fusion of inertial, optical flow, and airspeed measurements for uav navigation in gps-denied environments,' in Unmanned Systems Technology XI, vol. 7332. International Society for Optics and Photonics, 2009, p. 733202.

[32] M. Burri, M. Bloesch, Z. Taylor, R. Siegwart, and J. Nieto, "A framework for maximum likelihood parameter identification applied on mavs," Journal of Field Robotics, vol. 35, no. 1, pp. 5-22, 2018.

[33] V. Wüest, V. Kumar, and G. Loianno, "Online estimation of geometric and inertia parameters for multirotor aerial vehicles," in 2019 International Conference on Robotics and Automation (ICRA). IEEE, 2019, pp. 1884-1890.

[34] F. M. Mirzaei and S. I. Roumeliotis, "A kalman filter-based algorithm for imu-camera calibration: Observability analysis and performance evaluation," IEEE transactions on robotics, vol. 24, no. 5, pp. 1143 1156, 2008.

[35] J. Kelly and G. S. Sukhatme, "Visual-inertial sensor fusion: Localization, mapping and sensor-to-sensor self-calibration," The International Journal of Robotics Research, vol. 30, no. 1, pp. 56-79, 2011.

[36] D. Gorinevsky and S. Boyd, "Optimization-based design and implementation of multidimensional zero-phase iir filters," IEEE Transactions on Circuits and Systems I: Regular Papers, vol. 53, no. 2, pp. 372-383, 2006.

[37] M. Bloesch, M. Burri, S. Omari, M. Hutter, and R. Siegwart, "Iterated extended kalman filter based visual-inertial odometry using direct photometric feedback," The International Journal of Robotics Research, vol. 36, no. 10, pp. 1053-1072, 2017.

[38] M. Li and A. I. Mourikis, "High-precision, consistent ekf-based visualinertial odometry," The International Journal of Robotics Research vol. 32, no. 6, pp. 690-711, 2013.

[39] K. J. Åström, "Model uncertainty and robust control," Lecture Notes on Iterative Identification and Control Design, pp. 63-100, 2000.

[40] S.-C. Lee and H.-S. Ahn, "Sensorless torque estimation using adaptive kalman filter and disturbance estimator," in Proceedings of 2010 IEEE/ASME International Conference on Mechatronic and Embedded Systems and Applications. IEEE, 2010, pp. 87-92.

[41] E. S. Jones and S. Soatto, "Visual-inertial navigation, mapping and localization: A scalable real-time causal approach," The International Journal of Robotics Research, vol. 30, no. 4, pp. 407-430, 2011.

[42] A. Chiuso, P. Favaro, H. Jin, and S. Soatto, "Structure from motion causally integrated over time," IEEE transactions on pattern analysis and machine intelligence, vol. 24, no. 4, pp. 523-535, 2002.

[43] A. J. Davison, "Real-time simultaneous localisation and mapping with a single camera," in null. IEEE, 2003, p. 1403

[44] C. X. Guo, D. G. Kottas, R. DuToit, A. Ahmed, R. Li, and S. I. Roumeliotis, "Efficient visual-inertial navigation using a rolling-shutter camera with inaccurate timestamps." in Robotics: Science and Systems. Citeseer, 2014.

[45] T. Lee, K. Dunn, and C. Chang, "On observability and unbiased estimation of nonlinear systems," in System Modeling and optimization. Springer, 1982, pp. 258-266.

[46] R. Hermann and A. Krener, "Nonlinear controllability and observability," IEEE Transactions on automatic control, vol. 22, no. 5, pp. 728-740, 1977.
[47] K. W. Lee, W. S. Wijesoma, and J. I. Guzman, "On the observability and observability analysis of slam," in 2006 IEEE/RSJ International Conference on Intelligent Robots and Systems. IEEE, 2006, pp. 35693574.

[48] A. Martinelli, "State estimation based on the concept of continuous symmetry and observability analysis: The case of calibration," IEEE Transactions on Robotics, vol. 27, no. 2, pp. 239-255, 2011.

[49] S. Weiss, M. W. Achtelik, S. Lynen, M. Chli, and R. Siegwart, "Realtime onboard visual-inertial state estimation and self-calibration of mavs in unknown environments," in 2012 IEEE international conference on robotics and automation. IEEE, 2012, pp. 957-964.

[50] J. A. Hesch, D. G. Kottas, S. L. Bowman, and S. I. Roumeliotis, "Camera-imu-based localization: Observability analysis and consistency improvement," The International Journal of Robotics Research, vol. 33, no. 1 , pp. 182-201, 2014

[51] A. Martinelli, D. Scaramuzza, and R. Siegwart, "Automatic selfcalibration of a vision system during robot motion," in Proceedings 2006 IEEE International Conference on Robotics and Automation, 2006. ICRA 2006. IEEE, 2006, pp. 43-48.

[52] S. Puthusserypady, "Applied signal processing," Lecture Notes, Technical University of Denmark, 2018. [Online]. Available: http: //bme.elektro.dtu.dk/31610/notes/chap_8.pdf

[53] H. Stalford, "High-alpha aerodynamic model identification of t-2c aircraft using the ebm method," Journal of Aircraft, vol. 18, no. 10, pp. 801-809, 1981 .

[54] S. Ramachandran, H. Schneider, J. Mason, and H. Stalford, "Identification of aircraft aerodynamic characteristics at high angles of attack and sideslip using the estimation before modeling/ebm/technique," in Guidance and Control Conference, 1977, p. 1169.

[55] P. Martin and E. Salaün, "The true role of accelerometer feedback in quadrotor control," in 2010 IEEE international conference on robotics and automation. IEEE, 2010, pp. 1623-1629.

[56] J. P. Hespanha, Linear systems theory. Princeton university press, 2018.

[57] S. Weiss and R. Siegwart, "Real-time metric state estimation for modular vision-inertial systems," in 2011 IEEE international conference on robotics and automation. IEEE, 2011, pp. 4531-4537.

[58] H. Nijmeijer and A. Van der Schaft, Nonlinear dynamical control systems. Springer, 1990, vol. 175

[59] A. Isidori, Nonlinear control systems. Springer Science \& Business Media, 2013.

[60] A. Kurusa, Differential geometry. Typotex, 2014

[61] N. Hitchin, "Differentiable manifolds," Lecture Notes, Oxford University, 2012.

[62] R. M. Murray, Z. Li, S. S. Sastry, and S. S. Sastry, A mathematical introduction to robotic manipulation. CRC press, 1994.

[63] K. M. Lynch and F. C. Park, Modern Robotics. Cambridge University Press, 2017

[64] R. Kümmerle, G. Grisetti, H. Strasdat, K. Konolige, and W. Burgard, "g 2 o: A general framework for graph optimization," in 2011 IEEE International Conference on Robotics and Automation. IEEE, 2011, pp. 3607-3613.

[65] C. Hertzberg, R. Wagner, U. Frese, and L. Schröder, "Integrating generic sensor fusion algorithms with sound state representations through encapsulation of manifolds," Information Fusion, vol. 14, no. 1, pp. 57-77, 2013.

[66] S. Agarwal, K. Mierle, and Others, "Ceres solver," http://ceres-solver. org.

[67] F. Bullo and R. M. Murray, "Proportional derivative (pd) control on the euclidean group," in European control conference, vol. 2, 1995, pp. 1091-1097.

[68] F. Gustafsson, "Determining the initial states in forward-backward filtering," IEEE Transactions on signal processing, vol. 44, no. 4, pp. 988-992, 1996

[69] M. Faessler, A. Franchi, and D. Scaramuzza, "Differential flatness of quadrotor dynamics subject to rotor drag for accurate tracking of highspeed trajectories," IEEE Robotics and Automation Letters, vol. 3, no. 2, pp. 620-626, 2017 